\title{
Nonlinear plasmonic amplification via dissipative soliton-plasmon resonances
}

\author{
Albert Ferrando* \\ Departament d'Òptica, Interdisciplinary Modeling Group, InterTech, Universitat de València, València, Spain
}

(Received 8 November 2016; published 9 January 2017)

\begin{abstract}
In this contribution we introduce a strategy for the compensation of plasmonic losses based on a recently proposed nonlinear mechanism: the resonant interaction between surface plasmon polaritons and spatial solitons propagating in parallel along a metal/dielectric/Kerr structure. This mechanism naturally leads to the generation of a quasiparticle excitation, the so-called soliplasmon resonance. We analyze the role played by the effective nonlinear coupling inherent to this system and how this can be used to provide a mechanism of quasiresonant nonlinear excitation of surface plasmon polaritons. We will pay particular attention to the introduction of asymmetric linear gain in the Kerr medium. The unique combination of nonlinear propagation, nonlinear coupling, and gain give rise to a scenario for the excitation of long-range surface plasmon polaritons with distinguishing characteristics. The connection between plasmonic losses and soliplasmon resonances in the presence of gain will be discussed.
\end{abstract}

DOI: 10.1103/PhysRevA.95.013816

\section{INTRODUCTION}

High intensity electromagnetic fields are characteristic for a surface plasmon polariton (SPP) in metal/dielectric interfaces [1,2]. These high intensity fields are achieved nearby the metal, so that this region becomes a natural scenario for the presence of nonlinear effects. If the interface is constituted by a metal and a Kerr medium, the characteristics of the propagation of the SPP can be strongly modified by the presence of the nonlinearity. In such a case, the propagation constant of the SPP becomes dependent on the plasmon amplitude. These nonlinear effects on SPPs were studied long ago defining what is known as a nonlinear plasmon [3-6]. The high intensities in the vicinity of the metal are also responsible for a variety of nonlinear effects. When the confinement of the electromagnetic field takes place at a nanometric scale, as in plasmonic nanostructures, a panoply of these nonlinear effects are expected to be strongly enhanced [7-10].

The high value of SPP losses for a single metal/dielectric interface in the visible and infrared domains dramatically reduces the SPP propagation length to a few tens of microns, a value clearly insufficient for many practical applications. Strategies to increase the SPP propagation length are then necessary to overcome this important limitation (see [11] and references therein.) The use of more complex waveguiding geometries than a simple interface permits access to SPP modes with different spatial distributions. Some of these modes, those in which the mode amplitude is more localized in the dielectric than in the metal, present larger propagation lengths [12]. This is the case of the long-range surface plasmon polariton (LRSPP), the low-loss symmetric mode of a thin metal slab immersed between dielectrics with similar refractive indices [13-15]. This strategy can increase the propagation length up to a few centimeters. In order to compensate plasmonic losses even further, the addition of gain into the dielectric part of the waveguide has been a second step exploited in recent years by different groups [16-24]. In the previous context, the combination of nonlinear effects with gain in plasmonic waveguides appears as a natural consequence. Different

*Corresponding author: albert.ferrando@uv.es theoretical proposals of gain-assisted nonlinear waveguiding structures have been developed recently [25-31]. Theoretical tools to analyze periodic plasmonic waveguides and metamaterials with loss and gain have been also reported [32]. Our contribution in this article points out in the same direction although it presents important distinguishing features.

Our approach is based on the concept of soliplasmon resonance. A soliplasmon resonance can be understood as a quasiparticle combining a SPP mode with a spatial soliton as a result of its resonant or quasiresonant interaction during propagation along a metal/dielectric/Kerr (MDK) interface [33,34]. Although the plasmon-soliton term refers generically to hybrid nonlinear solutions involving metal and Kerr media and they were already studied during the 1980s [3-6], they do not necessarily deal with situations in which they exhibit a manifest resonant behavior between plasmon and soliton [25,35-46].

Mathematically, a plasmon-soliton solution of the nonlinear Maxwell's equations is formalized as a soliplasmon when it can be approximated by the so-called soliplasmon ansatz [47]. Physically, the soliplasmon ansatz represents a variational solution of nonlinear Maxwell's equations in which the plasmon and soliton components are distinguishable-thus spatially separated-and not strongly overlapping. The variational equations for the soliplasmon ansatz are rather simple in form since they correspond to two coupled oscillators: one linear (for the SPP variational amplitude) and the other nonlinear (for the soliton amplitude). However, the coupling presents a distinguishing feature: it is nonlinear and evanescent with the soliton amplitude $\left|C_{s}\right|$ and position $a$, i.e., $q \propto$ $\exp \left(-K\left|C_{s}\right| a\right)[33,47]$. Besides, it is nonsymmetric, which means that the soliton-to-plasmon coupling coefficient $q$ is generically much larger than the plasmon-to-soliton one $\bar{q}$ [47]. Although obtained explicitly for a MDK interface, the variational soliplasmon approach applies to more general 2D waveguiding structures in which the linear component can be a plasmonic mode (not just a SPP) and the nonlinear component can represent a nonlinear dielectric mode (not just a spatial soliton of an homogeneous medium). The particular characteristics of this nonlinear evanescent coupling makes the soliplasmon model different from previous approaches for similar metal/Kerr systems in which coupling terms turn 
out to be linear and symmetric [37,39]. The distinguishing properties of the nonlinear evanescent coupling was reported in [48,49], in which the soliplasmon model-for symmetric coupling - was mapped into that of a bosonic Josephson junction (BJJ). The dynamics of this nonlinear BJJ was proven to be-qualitatively and quantitativelydifferent from that of a standard linear coupling BJJ, thus indicating the crucial role played by the nonlinear evanescent coupling.

In this article we will analyze the profound effect that the addition of gain in the Kerr medium has in the propagation properties of the plasmonic component of a soliplasmon resonance. The content of the article is distributed as follows: in Sec. II we introduce the dissipative soliplasmon model; in Sec. III we construct the spin model associated to this model; in Secs. IV and V we obtain the stationary dissipative soliplasmon solutions of the spin model; in Sec. VI we present the phenomenon of critical gain linked to these solutions; and, finally, in Sec. VII we unveil how dissipative soliplasmons can act as mediators for strong nonlinear plasmonic amplification.

\section{SOLIPLASMON MODEL WITH LOSS AND GAIN}

The variational model for the nondissipative soliplasmon system was developed with detail in Ref. [47]. A summary of its main results is given in Appendix A. The variational model for conservative soliplasmons was obtained assuming that the propagation of a monochromatic quasistationary TM solution along a MDK structure such as in Fig. 1 can be approximated by the soliplasmon ansatz:

$$
E_{x}(x, z)=C_{p}(z) e_{p}(x)+C_{s}(z) \operatorname{sech}\left[\sqrt{\frac{\gamma}{2}}\left|C_{s}(z)\right|(x-a)\right],
$$

where $e_{p}(x)$ is a linear SPP solution of the MD interface, $C_{p}(z)$ and $C_{s}(z)$ are the two variational parameters, $a$ is the soliton position, and $\gamma=(3 / 4) \varepsilon_{0} c_{2}$ is the nonlinear coefficient of the Kerr medium. The other components of the TM mode, i.e., $H_{y}$ and $E_{z}$, can be obtained by using additional constraints valid under reasonable approximations [47]. In this first approximation the metal is considered to be an ideal conductor with $\operatorname{Im}\left(\varepsilon_{m}\right)=0$. However, for realistic lossy metals in which $\operatorname{Im}\left(\varepsilon_{m}\right) \neq 0$ the plasmon propagation constant becomes complex $\beta_{p}^{2}=\beta_{p}^{\prime 2}+i \beta_{p}^{\prime \prime 2}$. Consequently, the paraxial plasmon propagation constant also becomes complex $\mu_{p}=\mu_{p}^{\prime}+i \mu_{p}^{\prime \prime}$.

Besides the effect of metal losses generating the complex SPP plasmon propagation constant, it is possible to add lineal gain or loss in the Maxwell's equation description of the MDK structure in Fig. 1(a). This can be done mathematically by considering a modified complex permittivity function $\widetilde{\varepsilon}_{L}(x)=\varepsilon_{L}(x)+i \delta \varepsilon_{L}(x)$, where $\varepsilon_{L}(x)$ is the linear permittivity function of the MDK structure (in which we assume that metal losses are already included) and the imaginary part $\delta \varepsilon_{L}(x)$ provides the loss-gain profile introduced additionally in the structure by means of other lossy or active components. It is possible to incorporate into our variational model these gain-loss effects in a relatively simple manner if we assume that their transverse distribution is clearly spatially separated between the plasmon and soliton regions with small overlapping. In this way, additional loss-gain occurring in the SPP localization region, i.e., in the MD layers, would be given by a modified complex profile $\widetilde{\varepsilon}_{p}(x)=\varepsilon_{p}(x)+i \delta \varepsilon_{p}(x)$, where $\varepsilon_{p}(x)$ would be the permittivity profile of the MD structure (including metal losses) given by the standard expression in Eq. (A2) with complex $\varepsilon_{m}$. On the other hand, loss-gain in the soliton localization region would be analogously described by the modified permittivity function $\widetilde{\varepsilon}_{s}(x)=\varepsilon_{s}(x)+i \delta \varepsilon_{s}(x)$, defined in the Kerr medium. Taking the imaginary part $\delta \varepsilon_{L}(x)$ as a small perturbation of the unperturbed one $\varepsilon_{L}(x)$ permits one to calculate easily the first-order correction-which will be purely imaginary-in terms of the unperturbed values using standard perturbation theory. The first-order corrections to the
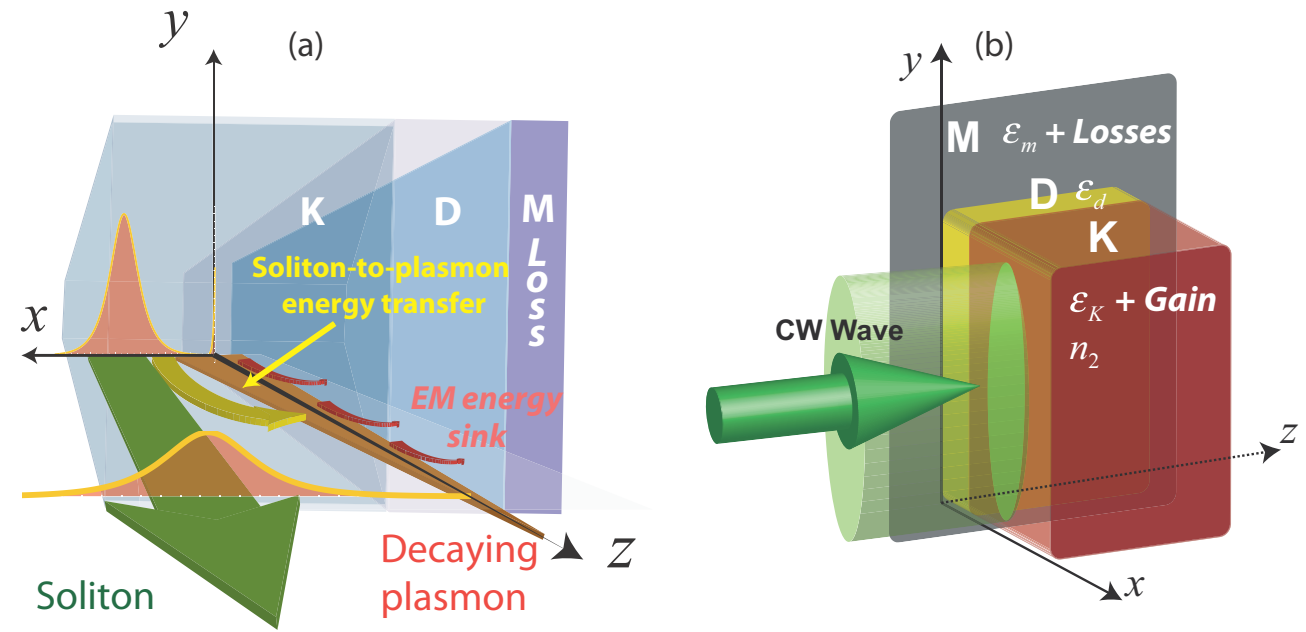

FIG. 1. (a) Scheme of the flux of EM energy in a soliplasmon structure with just metal losses. The SPP component is localized transversely ( $x$ axis) with a maximum at the MD interface $(x=0)$, whereas the spatial soliton component is centered at a certain distance $x=a$ from the MD interface within the Kerr medium. Both propagate in parallel to the MD and DK interfaces. (b) Characteristic MDK structure supporting dissipative soliplasmons in which gain is located in the Kerr medium in order to compensate for metal losses. 
propagation constants are given by [50]

$$
\begin{gathered}
\delta \beta_{p}^{\prime \prime 2}=\frac{1}{N_{p}} \int_{\mathbb{R}} d x \delta \varepsilon_{L}(x) e_{p}^{2}(x) \\
\approx \frac{1}{N_{p}} \int_{\Omega_{\mathrm{MD}}} d x \delta \varepsilon_{p}(x) e_{p}^{2}(x) \\
\delta \beta_{s}^{\prime \prime 2}=\frac{1}{N_{s}} \int_{\mathbb{R}} d x \delta \varepsilon_{L}(x) \operatorname{sech}^{2}\left[\sqrt{\frac{\gamma}{2}}\left|C_{s}\right|(x-a)\right] \\
\approx \frac{1}{N_{s}} \int_{\Omega_{K}} d x \delta \varepsilon_{s}(x) \operatorname{sech}^{2}\left[\sqrt{\frac{\gamma}{2}}\left|C_{s}\right|(x-a)\right],
\end{gathered}
$$

where we have used the spatial localization assumption on the loss-gain functions to determine the integration domains $\Omega_{\mathrm{MD}}$ $(x \leqslant d)$ and $\Omega_{K}(x>d)$ [47]. Therefore, the full propagation constants including the metal loss and the contribution introduced by extra gain or loss sources in the system are

$$
\begin{aligned}
& \beta_{p}^{2}=\beta_{p}^{\prime 2}+i\left(\beta_{p}^{\prime 2}+\delta \beta_{p}^{\prime \prime 2}\right), \\
& \beta_{s}^{2}=\beta_{s}^{\prime 2}+i \delta \beta_{s}^{\prime \prime 2} .
\end{aligned}
$$

The calculation of the complex paraxial propagation constants

$$
\begin{aligned}
& \mu_{p}=\mu_{p}^{\prime}+i \mu_{p}^{\prime \prime}, \\
& \mu_{s}=\mu_{s}^{\prime}+i \mu_{s}^{\prime \prime}
\end{aligned}
$$

is performed by means of Eqs. (A7) but using now the complex propagation constants (5). The real part of the soliton paraxial propagation constant depends nonlinearly on the soliton component $C_{S}$ :

$$
\mu_{s}^{\prime}\left(C_{s}\right)=\frac{k_{0} \gamma}{4 \varepsilon_{\mathrm{K}}^{1 / 2}}\left|C_{s}\right|^{2},
$$

where $\gamma$ is the nonlinear coefficient of the Kerr medium previously defined and $\varepsilon_{K}$ its real linear dielectric constant.

An analogous analysis holds also for the coupling coefficients $q$ and $\bar{q}$. The local permittivity functions $\Delta \varepsilon_{p}$ and $\Delta \varepsilon_{s}$, as defined in Eqs. (A10), pick up additional imaginary parts from the loss-gain function $\delta \varepsilon_{L}$ associated to the new total permittivity function $\widetilde{\varepsilon}_{L}(x)=\varepsilon_{L}(x)+i \delta \varepsilon_{L}(x)$. According to their definitions, the perturbed local permittivity functions are now

$$
\begin{aligned}
\triangle \widetilde{\varepsilon}_{p}(x) & =\widetilde{\varepsilon}_{L}(x)-\varepsilon_{p}(x)=\Delta \varepsilon_{p}(x)+i \delta \varepsilon_{L}(x), \\
\triangle \widetilde{\varepsilon}_{s}(x) & =\widetilde{\varepsilon}_{L}(x)-\varepsilon_{s}(x)=\Delta \varepsilon_{s}(x)+i \delta \varepsilon_{L}(x),
\end{aligned}
$$

where $\triangle \varepsilon_{p}$ and $\triangle \varepsilon_{s}$ would be given by Eqs. (A10) and correspond to an unperturbed MDK structure in which only $\varepsilon_{m}$ is complex (lossy metal). In this way, the first-order correction to the coupling coefficients will be given by the overlapping integrals:

$$
\begin{aligned}
& \delta q=\frac{k_{0}}{2 \varepsilon_{K}^{1 / 2} N_{p}} \int_{\mathbb{R}} d x e_{p}(x) \delta \varepsilon_{L}(x) \operatorname{sech}\left[\sqrt{\frac{\gamma}{2}}\left|C_{s}\right|(x-a)\right], \\
& \delta \bar{q}=\frac{k_{0}}{2 \varepsilon_{K}^{1 / 2} N_{s}} \int_{\mathbb{R}} d x e_{p}(x) \delta \varepsilon_{L}(x) \operatorname{sech}\left[\sqrt{\frac{\gamma}{2}}\left|C_{s}\right|(x-a)\right],
\end{aligned}
$$

in such a way that the whole coupling coefficients take the form

$$
\begin{aligned}
& \widetilde{q}=q+i \delta q=q^{\prime}+i \delta q^{\prime \prime}, \\
& \widetilde{\bar{q}}=\bar{q}+i \delta \bar{q}=\bar{q}^{\prime}+i \delta \bar{q}^{\prime \prime},
\end{aligned}
$$

where $q^{\prime}=\operatorname{Re}(\widetilde{q})$ and $\delta q^{\prime \prime}=\operatorname{Im}(\widetilde{q})$ and analogously for $\widetilde{\bar{q}}$. Note that both $q$ and $\delta q$ are complex so that they are not exactly the real and imaginary parts of $\widetilde{q}$. However, their imaginary parts are relatively small so that it is not a bad approximation to consider $q \approx q^{\prime}$ and $\delta q \approx \delta q^{\prime \prime}$.

In summary, the introduction of localized loss or gain in the MDK structure can be also analyzed using the same soliplasmon model in Appendix A but with complex propagation constants and couplings:

$$
\begin{aligned}
& -i \frac{d C_{p}}{d z}=\left(\mu_{p}^{\prime}+i \mu_{p}^{\prime \prime}\right) C_{p}+\left(q^{\prime}+i \delta q^{\prime \prime}\right) C_{s}, \\
& -i \frac{d C_{s}}{d z}=\left(\mu_{s}^{\prime}+i \mu_{s}^{\prime}\right) C_{s}+\left(\bar{q}^{\prime}+i \delta \bar{q}^{\prime \prime}\right) C_{p} .
\end{aligned}
$$

We will refer to this model as the dissipative soliplasmon model.

\section{SPIN MODEL}

In this article, we are interested in the search for solutions in which the intrinsic plasmon loss is compensated by the gain in the Kerr medium. In the absence of coupling the soliplasmon model equations (7) indicate that the plasmon and soliton parameters evolve as $C_{p}(z) \sim e^{i \mu_{p}^{\prime} z} e^{-\mu_{p}^{\prime \prime} z}$ and $C_{S}(z) \sim$ $e^{i \mu_{s}^{\prime} z} e^{-\mu_{s}^{\prime \prime} z}$, respectively. Thus, in order to make the loss-gain balance more explicit we write the imaginary parts of the paraxial propagation constants as $\mu_{p}^{\prime \prime}=k_{0} l$ and $\mu_{p}^{\prime \prime}=-k_{0} g$, where $l$ and $g$ are the positive-definite dimensionless loss and gain coefficients. Note that due to the lack of symmetry of the MDK structure these effective coefficients correspond to an asymmetric spatial distribution of loss and gain; thus it is expected that $l \neq g$ in the most general case. If we divide Eqs. (7) by $k_{0}$, all the coefficients of the system of differential equations becomes dimensionless. Besides, if we introduce the new propagation variable $\bar{z}=k_{0} z$, the differential operator becomes dimensionless as well. This procedure is equivalent to set $k_{0}=1$, so that $\mu_{p}, \mu_{s}, q$, and $\bar{q}$ become dimensionless and, in addition, the measurement of axial distances is done in units of $k_{0}^{-1}=\lambda / 2 \pi$ (from now on we maintain the notation of $z$ as the axial variable with this interpretation).

The system of coupled equations (7) admits the following matrix representation:

$$
-i \frac{d C}{d z}=H C,
$$

where $C=\left(C_{p}, C_{s}\right)^{\top}$ and

$$
H=\left[\begin{array}{cc}
\mu_{p} & q \\
\bar{q} & \mu_{s}
\end{array}\right]
$$

is the Hamiltonian of the system, where, in principle, all its elements are complex.

The matrix $H$ is non-Hermitian $\left(H \neq H^{\dagger}, H^{\dagger}\right.$ being the conjugate transpose or adjoint matrix of $H$ ) but it admits the 
following decomposition in Hermitian matrices:

$$
H=\Pi+i \Sigma,
$$

where

$$
\begin{aligned}
& \Pi=\frac{H+H^{\dagger}}{2}=\left[\begin{array}{cc}
\mu_{p}^{\prime} & q_{0} \\
q_{0}^{*} & \mu_{s}^{\prime}
\end{array}\right], \\
& \Sigma=\frac{-i}{2}\left(H-H^{\dagger}\right)=\left[\begin{array}{cc}
l & -i \Delta_{0} \\
i \Delta_{0}^{*} & -g
\end{array}\right],
\end{aligned}
$$

in which we have introduced the notation $q_{0} \equiv \frac{1}{2}\left(q+\bar{q}^{*}\right)$ and $\Delta_{0} \equiv \frac{1}{2}\left(q-\bar{q}^{*}\right)$. We see that $\Pi$ represents the Hermitian part of the Hamiltonian $H$. If $\Sigma$ were absent, the evolution of the system would be conservative- energy would be conservedand the norm of the vector $C$ would be preserved since the evolution operator $\exp (i \mathrm{~Hz})$ would be unitary. The matrix $\Sigma$ is responsible for the breaking of the Hermiticity of $H$ and, thus, of the unitary evolution of $C$.

Now we introduce the equivalent of the density matrix of a pure state $\rho=|C\rangle\langle C|$, which in matrix notation would be simply $\rho=C \cdot C^{\dagger}$, where the dot represents standard matrix multiplication. Our goal is to find the evolution equation for $\rho$ taking into account that $H$ is no longer Hermitian. In order to do so we need first to find the adjoint matrix equation associated to (8), which is simply

$$
i \frac{d C^{\dagger}}{d z}=C^{\dagger} H^{\dagger} .
$$

Using both equations it is straightforward to prove that

$$
-i \frac{d \rho}{d z}=H \rho-\rho H^{\dagger}=[\Pi, \rho]+i\{\Sigma, \rho\},
$$

where $[A, B]=A B-B A$ and $\{A, B\}=A B+B A$ are the commutator and anticommutator matrices, respectively. In order to convert the previous equation into an evolution equation for a spin model we resort to the standard decomposition of the $\rho$ matrix in terms of the identity and Pauli matrices $\left\{\tau_{0}, \tau_{1}, \tau_{2}, \tau_{3}\right\}$ (where $\tau_{0}=I$ ), which constitute a basis of $2 \mathrm{D}$ matrices:

$$
\rho=\sum_{i=0}^{3} S_{i}\left(\frac{\tau_{i}}{2}\right)
$$

An analogous decomposition applies to the $\Pi$ and $\Sigma$ matrices:

$$
\begin{aligned}
& \Pi=\sum_{j=0}^{3} \Omega_{j}\left(\frac{\tau_{j}}{2}\right), \\
& \Sigma=\sum_{j=0}^{3} \sigma_{j}\left(\frac{\tau_{j}}{2}\right) .
\end{aligned}
$$

Since $\rho, \Pi$, and $\Sigma$ are Hermitian matrices, all their components in the $\left\{\tau_{0}, \boldsymbol{\tau}\right\}$ basis are real. Taking into account the commutation and anticommutation algebra of Pauli matrices:

$$
\begin{aligned}
& {\left[\tau_{i}, \tau_{j}\right]=2 i \epsilon_{i j k} \tau_{k}, \quad i, j, k=1,2,3,} \\
& \left\{\tau_{i}, \tau_{j}\right\}=2 \delta_{i j} I, \quad i, j, k=0, \ldots, 3,
\end{aligned}
$$

one can readily prove the evolution equation for the fourdimensional spinlike vector $S=\left(S_{0}, \mathbf{S}\right)$ in terms of the four- dimensional vectors $\Omega=\left(\Omega_{0}, \Omega\right)$ and $\sigma=\left(\sigma_{0}, \boldsymbol{\sigma}\right)$ :

$$
\frac{d S_{0}}{d z}=-\sigma_{0} S_{0}-\sigma \cdot \mathbf{S}
$$

$$
\frac{d \mathbf{S}}{d z}=-\Omega \times \mathbf{S}-\sigma_{0} \mathbf{S}-S_{0} \boldsymbol{\sigma} .
$$

All these vector components can be found out of the elements of their generating matrices (10) by using proper projections. The algebra of $\left\{\tau_{0}, \boldsymbol{\tau}\right\}$ matrices provides the adequate projection operators by means of suitable tracing: $\Omega_{j}=\operatorname{Tr}\left[\Pi \tau_{j}\right]$ and $\sigma_{j}=\operatorname{Tr}\left[\Sigma \tau_{j}\right]$. Therefore,

$$
\begin{aligned}
& \boldsymbol{\Omega}=\left[\begin{array}{c}
q_{0}+q_{0}^{*} \\
i\left(q_{0}-q_{0}^{*}\right) \\
\mu_{p}^{\prime}-\mu_{s}^{\prime}
\end{array}\right]=\left[\begin{array}{c}
\bar{q}^{\prime}+q^{\prime} \\
\delta \bar{q}^{\prime \prime}-\delta q^{\prime \prime} \\
\mu_{p}^{\prime}-\mu_{s}^{\prime}
\end{array}\right], \\
& \boldsymbol{\sigma}=\left[\begin{array}{c}
-i\left(\Delta_{0}-\Delta_{0}^{*}\right) \\
\Delta_{0}+\Delta_{0}^{*} \\
l+g
\end{array}\right]=\left[\begin{array}{c}
\delta \bar{q}^{\prime \prime}+\delta q^{\prime \prime} \\
-\bar{q}^{\prime}+q^{\prime} \\
l+g
\end{array}\right],
\end{aligned}
$$

along with

$$
\begin{aligned}
\Omega_{0} & =\mu_{p}^{\prime}+\mu_{s}^{\prime}, \\
\sigma_{0} & =l-g .
\end{aligned}
$$

As it is well known, an identical procedure leads to the definition of the four-dimensional real spin vector $S=\left(S_{0}, \mathbf{S}\right)$ in terms of the original variational $2 \mathrm{D}$ complex vector $C=$ $\left(C_{p}, C_{s}\right)^{\top}$ :

$$
S=\left[\begin{array}{c}
C_{p}^{*} C_{s}+C_{s}^{*} C_{p} \\
i\left(C_{s}^{*} C_{p}-C_{p}^{*} C_{s}\right) \\
\left|C_{p}\right|^{2}-\left|C_{s}\right|^{2}
\end{array}\right]=\left[\begin{array}{c}
2\left|C_{p}\right|\left|C_{s}\right| \cos \phi_{s p} \\
-2\left|C_{p}\right|\left|C_{s}\right| \sin \phi_{s p} \\
\left|C_{p}\right|^{2}-\left|C_{s}\right|^{2}
\end{array}\right],
$$

where $\phi_{s p}=\phi_{p}-\phi_{s}$ is the relative phase between the plasmon and soliton components. Additionally,

$$
S_{0}=\left|C_{p}\right|^{2}+\left|C_{s}\right|^{2}=|\mathbf{S}| .
$$

Because of the latter constraint, the first evolution equation for $S_{0}$ in Eqs. (11) is not independent. It can be obtained from the second one for $\mathbf{S}$ by scalarly multiplying the latter by $\mathbf{S}$ and by taking into account the constraint $S_{0}=|\mathbf{S}|$.

It is interesting to remark here on the different nature and physical meaning of the $\boldsymbol{\Omega}$ and $\sigma$ four-vectors in the spin model represented by Eqs. (11). In the absence of the contribution of the $\sigma$ four-vector, the system becomes a standard model of a conservative spin system interacting with an external magnetic field $\boldsymbol{\Omega}$. This model is a well-known representation of a conservative quantum two-level system, in this case represented by the vector $|C\rangle=\left(C_{p}, C_{s}\right)^{\top}$. Note, however, that in our case, even in the conservative regime in which $\sigma_{0}=0$ and $\sigma=0$, both the original and the equivalent spin models are nonlinear in the sense that the soliton propagation constant $\mu_{s}$ as well as the couplings $q$ and $\bar{q}$ are functions of the soliton amplitude. Particularly, the nonlinear character of the couplings confers distinctive features on the dynamics of the system [48]. A nonzero value of the $\sigma=\left(\sigma_{0}, \boldsymbol{\sigma}\right)$ four-vector is generated when the Hamiltonian is non-Hermitian, and this fact can be produced either because its diagonal terms become complex 
$(l, g \neq 0)$ or because its off-diagonal terms are not conjugate of each other $\left(q^{*} \neq \bar{q}\right)$. In this way, the asymmetry in the coupling $(|q| \neq|\bar{q}|)$ appearing in the variational equations (A6) even in the absence of loss and gain-as demonstrated in Ref. [47] one expects $\bar{q} \ll q$ in realistic cases-is also responsible for the breaking of the Hermitian character of the dynamics. By simple inspection of the expressions for $\left(\sigma_{0}, \sigma\right)$ in Eqs. (12) we recognize that in the absence of gain and loss $(l=g=0)$ a zero value for its zero and third component is obtained $\left(\sigma_{0}=\sigma_{3}=0\right)$, whereas the "symmetry-conjugate" condition $\left(q^{*}=\bar{q}\right)$ ensures the vanishing of its other two components $\left(\sigma_{1}=\sigma_{2}=0\right)$. Thus the presence of a nonzero $\left(\sigma_{0}, \sigma\right)$ vector in the spin equations can be interpreted as a signature of the non-Hermiticity of the Hamiltonian for the variational vector $C$ caused by two different mechanisms: existence of dissipation and/or gain (when $l, g \neq 0$ ) and/or asymmetry in the conjugated coupling (when $q^{*} \neq \bar{q}$ ).

\section{STATIONARY SPIN STATES}

In the absence of dissipation and gain, stationary states of the soliplasmon model (A6) have been found previously for both symmetric [33,48] and asymmetric coupling [34]. These solutions are interpreted as bound states of a SPP and spatial soliton, the so-called soliplasmons, as they are characterized by a real propagation constant. This interpretation is confirmed by numerical simulations of full Maxwell's equations [34] and by a theoretical variational approach [47]. Stationary states of the soliplasmon model represent solutions of full vector nonlinear Maxwell's equations, i.e., they provide a variational approximation to a monochromatic and stationary 2D nonlinear wave. In this way, the soliplasmon can be considered, in turn, a soliton, in which diffraction and nonlinearity subtly cancel each other to generate a stationary state.

In Figs. 2(b) and 2(c) we provide some characteristic examples of the two families of soliplasmon solutions provided by the variational model (7) with the ansatz (1) in the absence of dissipation and gain. The so-called zero-soliplasmon solutions in Fig. 2(b) constitute a family characterized by a relative phase $\phi_{s p}=0$, whereas the $\pi$-soliplasmon family in Fig. 2(c) is characterized by a relative phase $\phi_{s p}=\pi$. According to the ansatz (1) and the definition of $e_{p}$ (A4), the peak value of the electric field at the metal interface is given approximately by $C_{p} / \varepsilon_{d}$, whereas the soliton peak value can be directly approximated by $C_{s}$. Since the variational soliplasmon model, when applied to a single MDK structure, has no free parameters we have chosen plausible values for the physical constants of the MDK structure. We take the Drude model value for the dielectric constant of silver at $\lambda=765 \mathrm{~nm}$ $\left(\varepsilon_{m}=-29.95\right), n_{d}=2.2$ for the linear dielectric, and we consider the chalcogenide glass $\mathrm{As}_{2} \mathrm{Se}_{3}$ as the Kerr medium with $n_{2}=3 \times 10^{-18} \mathrm{~m}^{2} / \mathrm{W}$ and $n_{K}=2.4$. The choice of $\mathrm{As}_{2} \mathrm{Se}_{3}$ is justified by the fact that observation of spatial solitons have been recently reported in slabs of this material with relatively moderate peak intensities of approximately $I_{0}=2 \mathrm{GW} / \mathrm{cm}^{2}$, which implies a peak value for the electric field of $E_{0} \sim 8 \times 10^{7} \mathrm{~V} / \mathrm{m}$ [51] [the electric field in Figs. 2(b) and 2(c) is normalized to this value] with an induced nonlinear refractive index of $\Delta n_{\mathrm{NL}} \sim 6 \times 10^{-6}$. The soliton position is $a=7.3 \mu \mathrm{m}$ and the width of the dielectric slab is $d=122 \mathrm{~nm}$. In any case, the purpose of the variational model is to provide a qualitative approximation to extant nonlinear solutions rather than an accurate description of them. It is in this sense that the values taken for the MDK structure intend to be close to realistic parameters.

When metal losses are naturally included in the model, soliplasmons cease to be stationary solutions since their plasmon component automatically "feels" the metal losses and it is exponentially attenuated during propagation. Such as pictorially reflected in Fig. 1(a), even in the presence of plasmonic losses, a nontrivial net exchange of energy from the soliton to the plasmon component of the soliplasmon
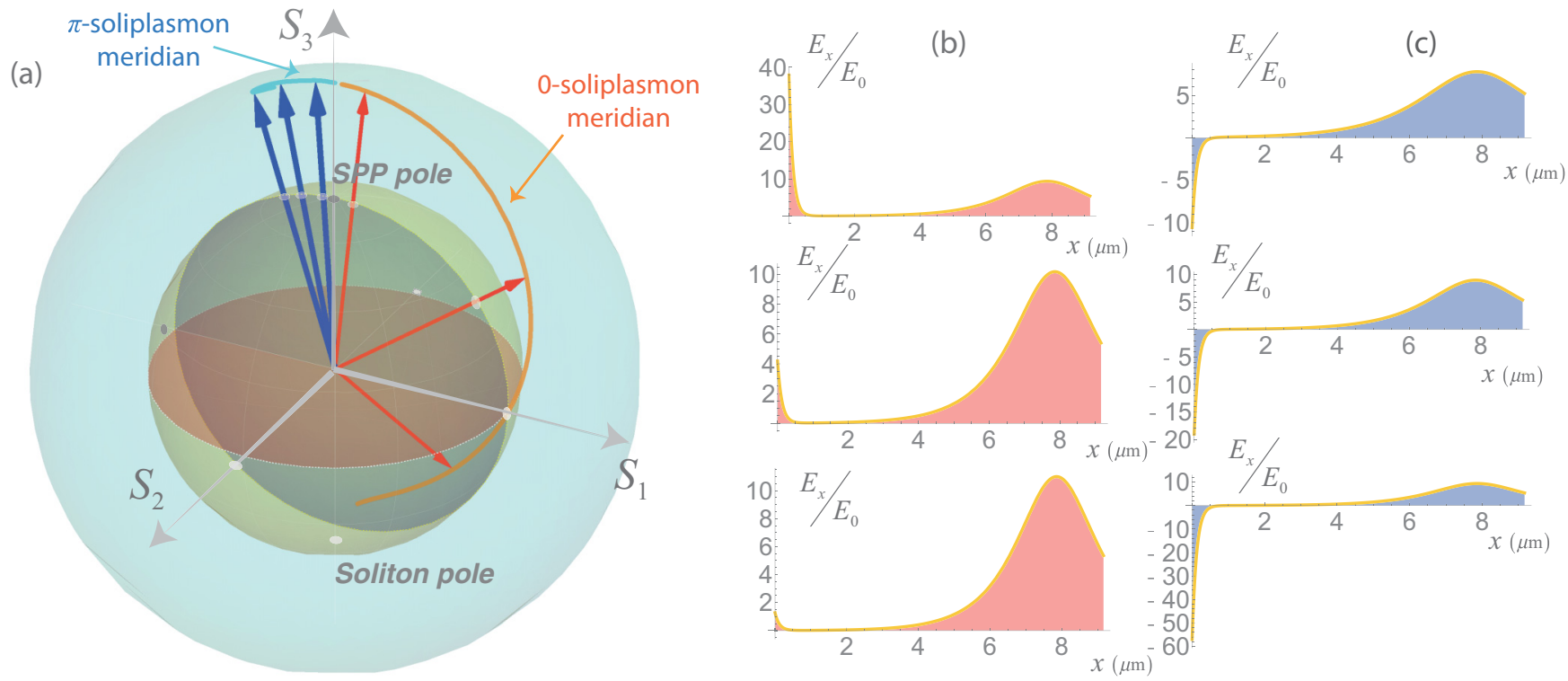

FIG. 2. Nondissipative soliplasmons: (a) 0 and $\pi$ soliplasmon families in the Earth-like Poincaré sphere (red arrows correspond to representative 0-soliplasmons solutions, blue arrows to $\pi$-soliplasmons ones); (b) intensity and phase of the three 0-soliplasmons solutions (red arrows) in the Poincaré sphere; (c) the same for the three $\pi$-soliplasmons solutions (blue arrows). 
occurs. As full simulations of nonlinear Maxwell's equations unveil, the pattern of energy exchange crucially depends on phase initial conditions in such a way attenuation of the plasmon component can be moderately compensated by energy pumped from the soliton component [34]. This feature is also successfully captured by the soliplasmon model when $l \neq 0$ (see also [34]). If partial compensation of plasmonic losses is achieved by soliton pumping when no gain is included in the system, a legitimate question to be considered is if total compensation can be fulfilled if we allow the system to obtain some gain in the region where the soliton is localized. In our generalized soliplasmon model this possibility is naturally incorporated since we precisely assumed the gain to be spatially localized in the nonlinear region. Therefore, we only need to let $l \neq 0$ and $g \neq 0$ in our loss and gain variational equations (7). The question now is whether the interplay between loss and gain will be able to generate new stationary solutions for which diffraction and nonlinearity will achieve again a new perfect balance.

Since we have proven in Sec. III that the loss and gain soliplasmon model (7) is equivalent to the spin model (11), the question of the perfect compensation of losses by soliton gain can be reformulated in terms of finding stationary spin solutions of these spin equations. The stationarity condition for the solutions of the loss and gain soliplasmon model (7) requires that both the modulus of the variational parameters $C_{p}$ and $C_{s}$ as well as their relative phase remain $z$ independent. In terms of its associated spin vector $S$ (13) this condition is equivalent to $d \boldsymbol{S} / d z=0$, which, according to the spin equation Eq. (11), implies the

$$
\boldsymbol{\Omega} \times \boldsymbol{S}+\sigma_{0} \boldsymbol{S}+S_{0} \boldsymbol{\sigma}=0
$$

or

$$
\boldsymbol{\Omega} \times \boldsymbol{n}+\sigma_{0} \boldsymbol{n}+\boldsymbol{\sigma}=0,
$$

where we have introduced the unitary vector $n \equiv S /|S|=$ $S / S_{0}$.

Remarkably, the previous equation for $\boldsymbol{n}$ admits the following explicit solution:

$$
\boldsymbol{n}=-\frac{1}{\sigma_{0}}\left(\frac{1}{\sigma_{0}^{2}+|\boldsymbol{\Omega}|^{2}}\right)\left[\sigma_{0}^{2} \boldsymbol{\sigma}+(\boldsymbol{\sigma} \cdot \boldsymbol{\Omega}) \boldsymbol{\Omega}+\sigma_{0}(\boldsymbol{\sigma} \times \boldsymbol{\Omega})\right] .
$$

Inasmuch as $\boldsymbol{n}$ is unitary, the unitarity condition $\boldsymbol{n} \cdot \boldsymbol{n}=1$ introduces the following important constraint on the $\left(\sigma_{0}, \boldsymbol{\sigma}\right)$ and $\left(\Omega_{0}, \boldsymbol{\Omega}\right)$ parameters:

$$
\sigma_{0}^{2}|\boldsymbol{\sigma}|^{2}+(\boldsymbol{\sigma} \cdot \boldsymbol{\Omega})^{2}=\sigma_{0}^{2}\left(\sigma_{0}^{2}+|\boldsymbol{\Omega}|^{2}\right)
$$

This constraint is a necessary condition to be fulfilled by the parameters of the spin model for the existence of the solution (15). It is a highly remarkable feature that this constraint is absent in the conservative case for which $\sigma_{0}=0$ and $\sigma=0$.

In order to illustrate the relevance of the constraint (16) it is instructive to approach the conservative regime by taking the limit $\sigma=\left(\sigma_{0}, \sigma\right) \rightarrow 0$ in Eqs. (15) and (16). We easily find that

$$
\begin{aligned}
\boldsymbol{n} & =-\frac{(\boldsymbol{\sigma} \cdot \boldsymbol{\Omega}) \boldsymbol{\Omega}}{\sigma_{0}|\boldsymbol{\Omega}|^{2}}+O(\sigma), \\
(\boldsymbol{\sigma} \cdot \boldsymbol{\Omega}) & = \pm \sigma_{0}|\boldsymbol{\Omega}|+O(\sigma)^{3},
\end{aligned}
$$

respectively. Therefore, by incorporating the approximated constraint in the equation for $\boldsymbol{n}$, we get

$$
\boldsymbol{n}=\mp \frac{\boldsymbol{\Omega}}{|\boldsymbol{\Omega}|}+O(\sigma)
$$

This is the correct solution in the conservative case, in which the stationary solution corresponds to a spin perfectly aligned with the external magnetic field $\boldsymbol{\Omega}$. As we can see, when taking the conservative limit the correct result is obtained only when one properly complements the solution with the constraint (16). On the other hand, as already emphasized, the conservative solution (17)—unlike the nonconservative spin solution (15) - automatically satisfies the unitarity condition $\boldsymbol{n} \cdot \boldsymbol{n}=1$ independent of the value of the parameters of the spin model.

Another interesting limit is the one corresponding to nondissipative solutions $(l=g=0)$ in the presence of asymmetric coupling $\left(q^{*} \neq \bar{q}\right)$ implying the non-Hermiticity of the Hamiltonian. If we neglect metal losses, this situation corresponds to the more common case of conservative soliplasmons for which the soliton-to-plasmon coupling is considerably larger than the plasmon-to-soliton one, and, therefore, coupling is clearly asymmetric [34,47]. According to Eqs. (12), in this situation we wish to take the $\sigma_{0} \rightarrow 0$ limit-keeping $\sigma$ finite-in the general solution (15) and in the constraint (16). A similar analysis to the previous one for the full-conservative case leads us to the following expression for the unitary spin vector $\boldsymbol{n}$ of nondissipative asymmetrically coupled solutions:

$$
\boldsymbol{n}=\frac{1}{|\boldsymbol{\Omega}|^{2}}\left[\mp\left(|\boldsymbol{\Omega}|^{2}-|\boldsymbol{\sigma}|^{2}\right)^{1 / 2} \boldsymbol{\Omega}-\boldsymbol{\sigma} \times \boldsymbol{\Omega}\right],
$$

together with the constraint for the spin model parameters $\boldsymbol{\sigma} \cdot \boldsymbol{\Omega}=0$. Note that the previous expression reduces to the conservative one (17) when we take the Hermiticity limit $\sigma \rightarrow$ 0 .

\section{STATIONARY DISSIPATIVE SOLIPLASMONS AND THE "GOLDEN CONSTRAINT"}

The previous analysis has no explicit reference to the dependence of the vectors $\left(\sigma_{0}, \boldsymbol{\sigma}\right)$ and $\boldsymbol{\Omega}$ on the parameters or our original dissipative soliplasmon model (7). In this sense, its validity goes beyond the specificities of our model describing soliplasmon dynamics. However, as we will see next, the specific properties of the dissipative soliplasmon model are important. The set of equations (12) provide the link between the generic spin model parameters and the matrix elements of the soliplasmon Hamiltonian (9). In order to understand the peculiar properties of the spin model of dissipative soliplasmons one has to keep in mind that all the coefficients of the Hamiltonian (9) except $\mu_{p}$ are nonlinear functions of the $z$-dependent soliton variational parameter $C_{s}(z)$.

\section{A. "Golden constraint" for nonlinear solutions}

We have stressed already in Sec. IV the importance of the constraint (16) to determine a legitimate spin solution. In terms of our original soliplasmon model this constraint 
becomes a condition to be fulfilled also by the parameters of the soliplasmon Hamiltonian (9). By substituting the values of the $\left(\sigma_{0}, \sigma\right)$ and $\left(\Omega_{0}, \boldsymbol{\Omega}\right)$ vectors in the constraint (16) using Eqs. (12) we obtain that the coefficients of the dissipative soliplasmon model (7) have to fulfill the following constraint:

$$
\begin{aligned}
& {\left[(g+l)\left(\mu_{p}^{\prime}-\mu_{s}^{\prime}\right)-\left(\delta \bar{q}^{\prime \prime}-\delta q^{\prime \prime}\right)\left(\bar{q}^{\prime}-q^{\prime}\right)\right]^{2}} \\
& \quad-(g-l)^{2}\left[-4 g l+\left(\mu_{p}^{\prime}-\mu_{s}^{\prime}\right)^{2}\right. \\
& \left.+4 q^{\prime} \bar{q}^{\prime}+\left(\delta \bar{q}^{\prime \prime}-\delta q^{\prime \prime}\right)^{2}\right]=0 .
\end{aligned}
$$

Since we are dealing with the imaginary part $\varepsilon_{L}^{\prime \prime}(x)$ as a small perturbation, the imaginary parts of the couplings $\delta q^{\prime \prime}$ and $\delta \bar{q}^{\prime \prime}$ have to be considered, in turn, small perturbation of their real parts $q^{\prime}$ and $\bar{q}^{\prime}$. On the other hand, the variational equations of the soliplasmon model were derived under the weak-coupling condition, so the coupling coefficients $q$ and $\bar{q}$ were assumed to be small with respect to the propagation constants $\mu_{p}$ and $\mu_{s}$. Consequently, we can further simplify Eq. (19) by keeping only the leading-order terms in both approximations, which in this context implies neglecting the imaginary parts of the couplings $\delta q^{\prime \prime}$ and $\delta \bar{q}^{\prime \prime}$. In this way we obtain a simple form of the constraint given by

$$
\begin{aligned}
& g l\left\{(g-l)^{2}+\left[\mu_{p}^{\prime}-\mu_{s}^{\prime}\left(C_{s}\right)\right]^{2}\right\}-(g-l)^{2} q^{\prime}\left(C_{s}\right) \bar{q}^{\prime}\left(C_{s}\right) \\
& \quad=0,
\end{aligned}
$$

where we have introduced the dependence on the soliton variational parameter $C_{s}$ explicitly.

Written in the form (20) it becomes clear the essential role played by the constraint equation, either in its simplified or general form, in the determination of the nonlinear solutions of a dissipative soliplasmon. Indeed, when all the nonvariational parameters of the problem are given, the constraint equation define whether a solution can exist or not. The constraint is nothing but a nonlinear equation for the modulus of the soliton parameter $\left|C_{s}\right|$. Specifically for the MDK structure under consideration, if we provide as an input its optical complex coefficients $\varepsilon_{m}, \varepsilon_{d}, \varepsilon_{K}$, the nonlinear index $n_{2}$, the width $d$, and the soliton position $a$, we can compute all terms in the constraint using the expressions given in the Appendix Aincluding the loss and gain coefficients $l$ and $g$-except the unknown value of $\left|C_{s}\right|$. Since this constraint is a necessary condition for a stationary spin solution as given in Eq. (15) to exist, if such a solution for $\left|C_{s}\right|$ cannot be found, we can state that the corresponding dissipative soliplasmon solution does not exist either. On the contrary, if one solution $\left|C_{s}\right|$ of the constraint is found we can univocally construct its associated spin $S$ and variational $C$ vectors. Once the value of $\left|C_{s}\right|$ is fixed by the constraint in terms of the system parameters, all the nonlinear coefficients $\mu_{s}^{\prime}, q$, and $\bar{q}$ depending on it are likewise fixed. It is interesting to emphasize that, due to the nonlinear character of the constraint (20), it is possible to find more than one solution for $\left|C_{s}\right|$.

Due to the paramount importance of the nonlinear constraint (20)_or (19)—for finding dissipative soliplasmon solutions we shall refer to it as the "golden constraint." Remarkably, the golden constraint only exists in the presence of dissipation. When we set $l=g=0$ in it, the constraint disappears and, therefore, no restriction is exerted on the nonlinear solutions of the problem. In the same way that the existence of conservative spatial solitons is rooted in the compensation between diffraction and nonlinearity, the golden constraint can be envisaged as the additional balance condition between losses and gain that a dissipative soliton has to fulfill in order to exist [52]. Unlike in the conservative case, in which soliton solutions constitute a continuous family, the golden constraint permit only a discrete number of them.

\section{B. Spin solution of a stationary dissipative soliplasmon}

We are now in conditions to find an explicit expression for a spin solution of a stationary dissipative soliplasmon. First, we substitute in the general stationary spin solution (15) the explicit expressions of the $\left(\sigma_{0}, \boldsymbol{\sigma}\right)$ and $\left(\Omega_{0}, \boldsymbol{\Omega}\right)$ vectors in terms of the coefficients of the dissipative soliplasmon model as given in Eqs. (12). Secondly, we implement the golden constraint (20) explicitly in the solution by eliminating the dependence on the propagation constant detuning $\mu_{p}^{\prime}-\mu_{s}^{\prime}$ in favor of a dependence on $l, g, q$, and $\bar{q}$. The result is

$$
\boldsymbol{n}=\left[\begin{array}{c}
2 \operatorname{sgn}(l-g)(g q+l \bar{q})^{-1}[(q \bar{q}-g l) g l]^{1 / 2} \\
-2 g l(g q+l \bar{q})^{-1} \\
-1+2 g q(g q+l \bar{q})^{-1}
\end{array}\right]
$$

We stress again that the problem of finding a dissipative soliplasmon solution is univocally solved once we have determined the specific value of $\left|C_{s}\right|$ that satisfies the golden constraint. This can be seen clearly in the previous expression, in which once $\left|C_{s}\right|$ is given $q$ and $\bar{q}$ are determined and the spin solution is fixed. The particular form of (21) is, however, not unique since we can implement the golden constraint differently from what we did to obtain this solution. We could have chosen to eliminate other parameter rather than the detuning. In that case the explicit form of $\boldsymbol{n}$ in Eq. (21) would look different although both expressions would correspond to the same solution.

The spin solution $\boldsymbol{n}$ in Eq. (21) is a unit vector, for which $S_{0}=|\boldsymbol{n}|^{2}=1$. Therefore, it does not contain information about the norm of the variational vector $C$, which is given by the zero component of the spin vector $S_{0}=\left|C_{p}\right|^{2}+\left|C_{s}\right|^{2}$. In order to define the spin solution of a dissipative soliplasmon completely we need to give an explicit expression of $S_{0}$ in terms of known parameters. From the definitions of $S_{0}$ and $S_{3}$ in Eq. (13), we immediately obtain

$$
S_{0}=\frac{2\left|C_{s}\right|^{2}}{1-n_{3}} .
$$

This expression together with the unit vector (21) define completely the spin solution of a dissipative soliplasmon since $\boldsymbol{S}=S_{0} \boldsymbol{n}$.

The Poincaré or Bloch sphere is a common way of representing the spin vector associated to a two-level quantum system. We will use it to represent soliplasmon states given by the previous spin equations. Inasmuch as Eq. (22) points out that soliplasmon states represented by spin vectors with arbitrarily large norm $S_{0}$ can exist (if $n_{3} \rightarrow 1$ ), we will extend the Poincare sphere in order to guarantee the visualization of these states. We introduce the "Earth-like" Poincaré sphere concept, which maps the infinite existence domain of $S_{0}$, 
given by $[0, \infty[$, into a finite one, $[0, \varphi]$, where $\varphi \approx 1.618$ is the golden ratio, to facilitate this visualization. Details of this construction are given in Appendix B. By definition, the Earth-like Poincaré sphere is defined by two regions: the inner sphere (the Earth's sphere), characterized by the transformed radius $\bar{S}_{0} \in[0,1]$, and the outer shell given by the domain $\left.\bar{S}_{0} \in\right] 1, \varphi[$ (the "atmospheric" layer.) All spins whose end point lies on the "atmosphere" layer will have $S_{0}>1$. The main advantage of this representation is that existing states with infinitely large norm approaching the $S_{0} \rightarrow \infty$ limit will appear represented by points infinitely close to the atmosphere's outer surface with radius $\varphi$ (i.e., $\bar{S}_{0} \rightarrow \varphi$ ).

As a first example of its use, we provide in Fig. 2(a) the spin representation of the nondissipative 0-soliplasmons (red arrows) and $\pi$-soliplasmons (blue arrows) of Figs. 2(b) and 2(c). The spin vectors for these solutions are given by Eqs. (18) and (22). Nondissipative soliplasmons are restricted to lie on the $S_{2}=0$ section of the sphere since its relative phase is either 0 or $\pi$. The 0 - and $\pi$-soliplasmon families form a continuum of solutions, which are represented in this figure by the orange and light blue curves, respectively. However, the norm of these solutions change with $C_{s}$, as clearly visualized in Fig. 2(a). The "Earth surface" has unity radius and it has been selected in order that soliplasmons with $S_{3}=0$, i.e., with $C_{p}=C_{s}$, lie on it. The north pole of the unit sphere is given by the plasmonic state $C_{p}=1$ and $C_{s}=0$. However, both the 0 - and $\pi$-soliplasmon curves tend to the north pole of the outer sphere indicating that for nondissipative soliplasmons these asymptotic states have infinitely large plasmonic component $\left(C_{p} \rightarrow \infty\right)$. An analogous interpretation could be given to the southern pole with increasingly solitonic states.

\section{CRITICAL GAIN}

We will use the Earth-like Poincaré sphere also to represent dissipative soliplasmons given by the spin vector defined by Eqs. (21) and (22). The main difference with respect to the nondissipative case is certainly the existence of the golden constraint (20) (we will focus on the simple version), which restricts the number of potential solutions to a discrete set instead of a continuous family. The number of solutions is determined by the finite number of roots in the $C_{s}$ variable given by Eq. (20). In order to have an idea of this number, it is convenient to rewrite (20) in the form

$$
q^{\prime}\left(C_{s}\right) \bar{q}^{\prime}\left(C_{s}\right)=g l\left\{1+\left[\frac{\mu_{p}^{\prime}-\mu_{s}^{\prime}\left(C_{s}\right)}{g-l}\right]^{2}\right\} .
$$

It can be checked that the product $q^{\prime} \bar{q}^{\prime}$ behaves approximately as $k_{1}\left|C_{s}\right| \exp \left(-k_{2}\left|C_{s}\right|\right)$, so that it presents the characteristic shape shown by the dashed curve in Fig. 3. In this representation we use the same parameters for the MDK structure as in the conservative case but taking now a realistic value for the complex silver dielectric constant $\left(\varepsilon_{m}=\right.$ $-34.0+1.8 i$ at $\lambda=870 \mathrm{~nm}$ ), which determines a SPP loss dimensionless coefficient of $l=1.05 \times 10^{-2}$ (corresponding to $l=758 \mathrm{~cm}^{-1}$, in physical units). The function at the righthand side of Eq. (23) is a slowly monotonically increasing function of $C_{s}$ parametrized by the gain coefficient $g$ (solid line in Fig. 3). The crossing points are the roots of the golden constraint fixing the allowed values of $C_{S}$ for the permitted dissipative soliplasmon solutions. The smooth gradient of the right-hand side function in Eq. (23) can be considered a general feature caused by the soft modulation of the induced nonlinear refractive index with the soliton amplitude for
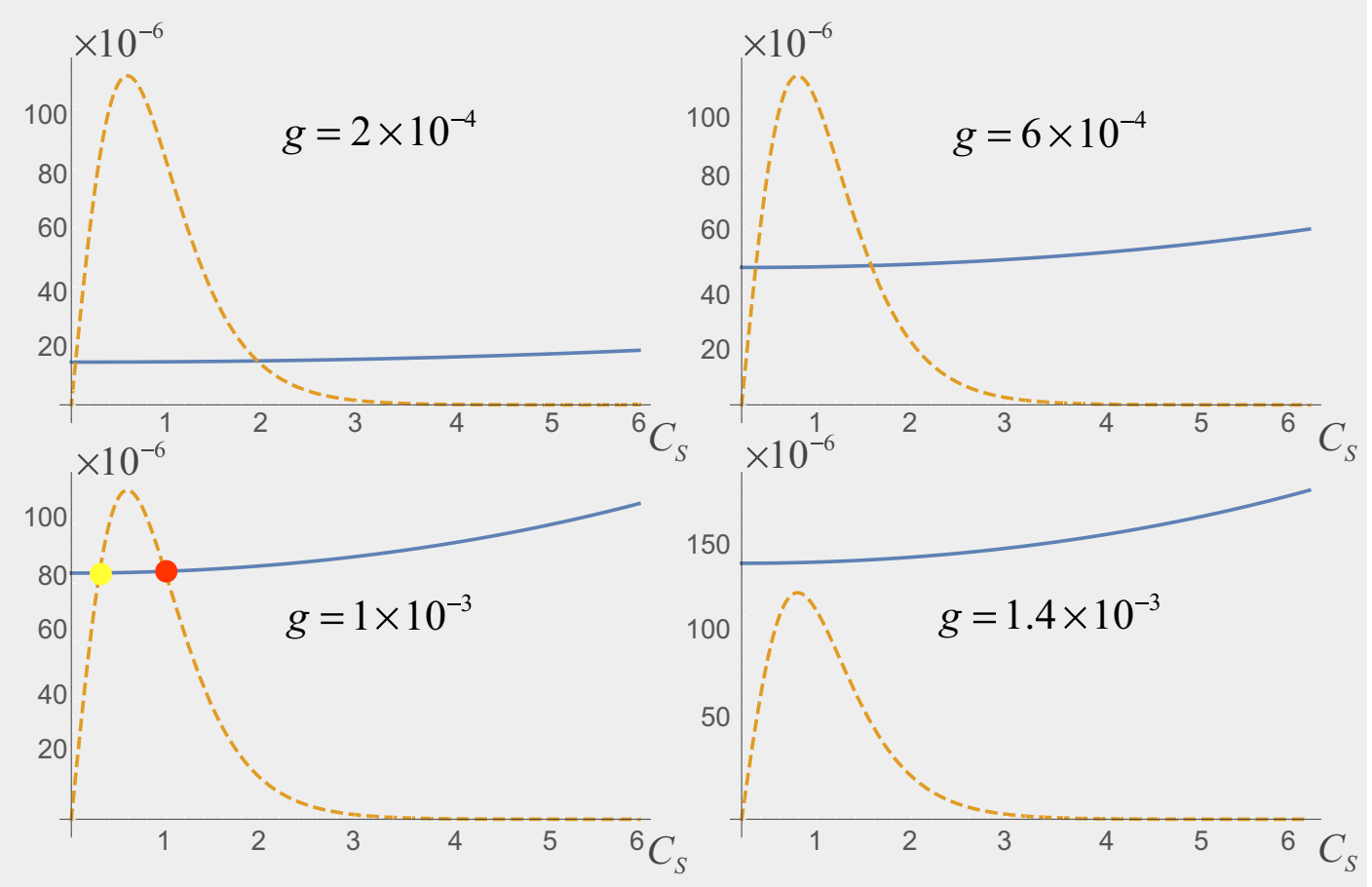

FIG. 3. Representation of the "golden constraint" for increasing values of the gain coefficient $g$ for a given loss coefficient of $l=1.05 \times 10^{-2}$ $\left(l=758 \mathrm{~cm}^{-1}\right.$, in physical units). 
plausible intensities in realistic nonlinear materials, due to the small value of the nonlinear coefficient $\gamma$. The sign of the slope depends on the relative sign between the SPP and soliton paraxial propagation constants.

The nonlinear character of the golden constraint determines the existence of a critical value for the gain. Due to the peculiar nonlinear dependence of $q^{\prime} \bar{q}^{\prime}$ on $\left|C_{s}\right|$, as compared to the smoothness of the dependence on $\left|C_{s}\right|$ of soliton propagation constant $\mu_{s}^{\prime}$, the typical scenario for crossing points is the one depicted in Fig. 3. As we increase $g$, the monotonically increasing right-hand side of Eq. (23) (solid line in Fig. 7) takes a larger value at the origin. Thus two crossing points are granted to exist until the solid line intersects the $q^{\prime} \bar{q}^{\prime}$ function at its maximum, something that occurs at a critical value of $g=g_{c}$. Consequently, for values of $g$ below $g_{c}$ there exist two stationary dissipative soliplasmon solutions. As $g$ tends to $g_{c}$ the two solutions become increasingly more similar in such a way that they become degenerate at $g=g_{c}$. The solution at $g=g_{c}$ is unique, and no stationary dissipative soliplasmon solution exists for $g>g_{c}$. We will refer to $g_{c}$ as the critical gain.

In the left-bottom side of Fig. 4 we represent in the Earthlike Poincaré sphere the two spins, denoted as $\boldsymbol{S}_{a}$ and $\boldsymbol{S}_{b}$, associated to the pair of soliplasmon solutions obtained from the roots of the golden constraint with gain coefficient $g=$ $1 \times 10^{-3}\left(g=72 \mathrm{~cm}^{-1}\right.$, in physical units) in Fig. 3. In this case, the Earth surface with unity radius has been chosen in such a way the purely solitonic soliplasmon, i.e., the state with $C_{p}=0$ and $C_{s}=E_{0}$, lies on it. Thus, by construction, the spin state $\left(0,0,-E_{0}^{2}\right)$ is the state whose norm sets the reference for all the states and it will represented by $S=(0,0,-1)$ in the Earth-like Poincaré sphere. The reason for taking this state as a reference will become clear when we analyze propagation in the next section. In general, $E_{0}$ is an arbitrary reference value. Here, as in the previous section and for the sake of comparison with realistic situations, we choose it to be $E_{0}=8 \times 10^{7} \mathrm{~V} / \mathrm{m}$, which approximately corresponds to the soliton peak value for realistic experiments in chalcogenide materials [51].

Dissipative soliplasmons qualitatively differ from the soliplasmon of the nondissipative system $(l=g=0)$ not only because they constitute a discrete set of two solutions instead of a family-compare Fig. 2 and the $\boldsymbol{S}_{a}$ and $\boldsymbol{S}_{b}$ solutions in Figs. 4(a) and 4(b) - but also because they are not restricted to lie in the $S_{2}=0$ section of the Earth-like Poincaré sphere. Therefore, they are neither 0 nor $\pi$ soliplasmons, as clearly visualized in Fig. 4 by the two spins $\boldsymbol{S}_{a}$ and $\boldsymbol{S}_{b}$. The loss and gain balance expressed in the golden constraint requires the stationary soliplasmon solutions to present a nontrivial relative phase to achieve perfect compensation. In our particular case, both solutions present a very similar relative phase close to $-\pi / 8$, as visualized in the Earth-like Poincaré sphere of Fig. 4, and thus an almost identical phase profile, as shown in Fig. 4. In our particular case, both spin solutions are very close to the SPP pole and to each other thus indicating they have large SPP components and similar shapes. The fact that dissipative soliplasmons with large SPP component exist is important for plasmonic nonlinear amplification, as we will see in the next section.

The stability mechanism behind these dissipative soliplasmon solutions is, in fact, very robust, as our simulation in Fig. 5 unveils. Our choice of the initial condition to demonstrate plasmonic amplification during propagation can be also considered as a stability check. Our initial purely solitonic state $S^{\text {in }}=(0,0,-1)$ can be taken as a perturbation of the final soliplasmon state $S_{a}$ (yellow arrow) by writing $\boldsymbol{S}^{\text {in }}=\boldsymbol{S}_{a}+\Delta \boldsymbol{S}_{a}$. It is clear in the Earth-like Poincaré sphere

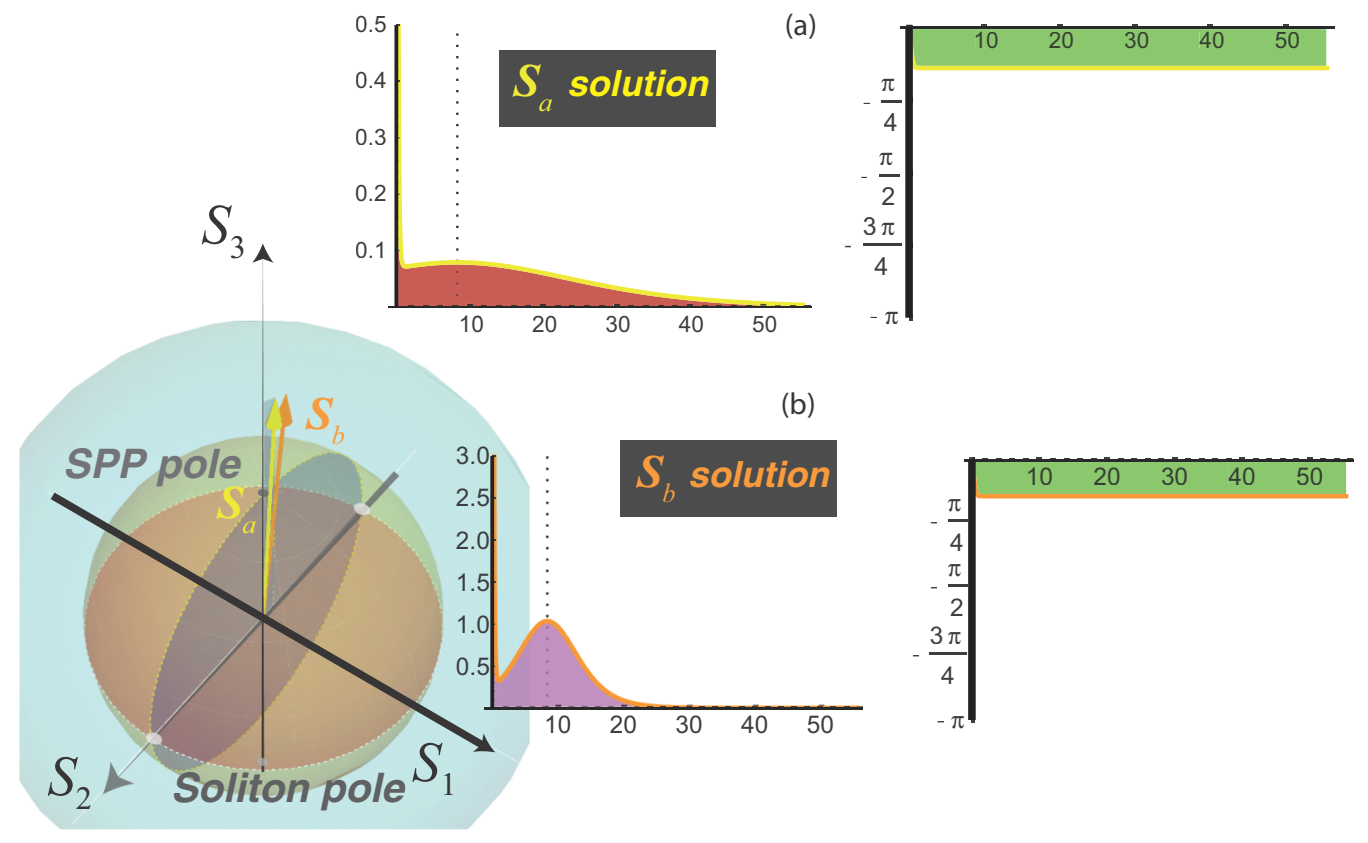

FIG. 4. Pair of dissipative soliplasmons in the "Earth-like" Poincaré sphere corresponding to the configuration with $g=1 \times 10^{-3}(g=$ $72 \mathrm{~cm}^{-1}$, in physical units) in Fig. 3: $\boldsymbol{S}_{a}$ (yellow arrow) solution with $C_{s}=0.295 ; \boldsymbol{S}_{b}$ (orange arrow) solution with $C_{s}=0.848$. Inset: intensity and phase profiles of the corresponding two dissipative soliplasmon solutions represented in the Earth-like Poincaré sphere. (a) $\boldsymbol{S}_{a}$ yellow arrow solution; (b) $S_{b}$ orange arrow solution. 


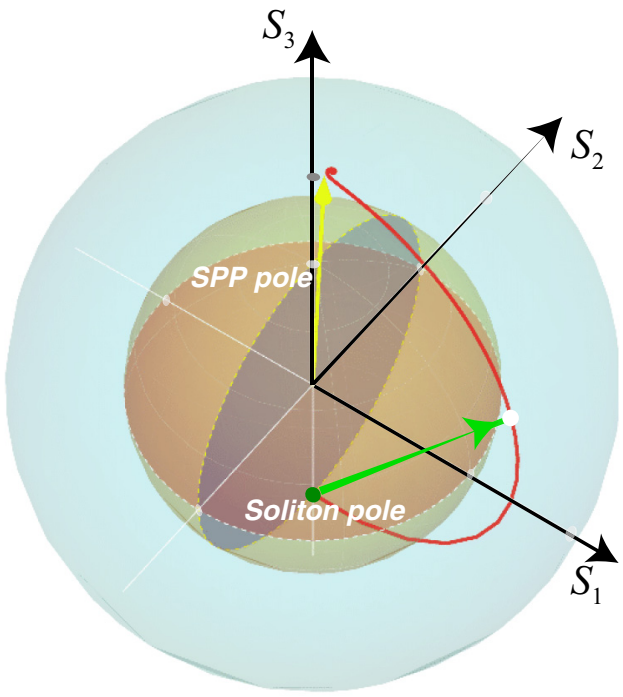

FIG. 5. Nonlinear amplification of a surface plasmon in the Earth-like Poincaré sphere. A purely solitonic input state (green dot) with no plasmonic component at the South pole evolves into a dissipative soliplasmon output state (yellow arrow) with a high plasmon component located near the north pole well above the Earth surface. Trajectory "takes off" from the soliton pole of the Earth surface into the "atmospheric" shell swiftly reaching a point of maximum height and amplification (white spot), then descending smoothly to reach the final output dissipative soliplasmon state.

that, since $\boldsymbol{S}_{a}$ is a "highly plasmonic" state-very close to the north pole- the perturbation $\Delta \boldsymbol{S}_{a}$ is huge since the initial state $S^{\text {in }}$ is located at the south pole. Despite this fact, the dissipative soliplasmon solution $\boldsymbol{S}_{a}$ acts as an attractor for $\boldsymbol{S}^{\text {in }}$ converting a state with no plasmonic component at all into a state with a large relative plasmonic component. On the one hand, the intermediate state of maximum amplification (white spot in Fig. 5) is not a stable solution; it is also belongs to the attractor basin of the stationary dissipative soliplasmon $\boldsymbol{S}_{a}$. The second solution $\boldsymbol{S}_{b}$ is, unlike $\boldsymbol{S}_{a}$, highly unstable. Numerical simulation of the evolution of a perturbed $\boldsymbol{S}_{b}$ spin state indicates that this state-even for perturbations as small as a $1 \%$ - can move very far away from its initial value or evolve into the stable spin $\boldsymbol{S}_{a}$.

\section{NONLINEAR AMPLIFICATION}

The existence of dissipative soliplasmons in a MDKD structure with gain in the Kerr medium provides an original mechanism for the amplification of surface plasmon polaritons. If a dissipative soliplasmon is the asymptotic stationary state of an initial state with smaller plasmon component, then amplification of the SPP signal occurs. The soliplasmon model and its spin representation provides a useful scenario to describe this mechanism. Using the soliplasmon ansatz (1),we can characterize the SPP amplification property in simple terms as

$$
\left|C_{p}^{\text {in }}\right|<\left|C_{p}^{\text {out }}\right|
$$

In our spin model, the output state with plasmon component $C_{p}^{\text {out }}$ will be represented by a dissipative soliplasmon spin
$S^{\text {out }}$ in the Earth-like Poincare sphere. At the same time, the states verifying the condition (24) will constitute in the Earthlike Poincaré sphere the domain $\boldsymbol{\Omega}_{\mathrm{amp}}\left(\boldsymbol{S}^{\text {out }}\right)$ of initial states that can be potentially amplified into $\boldsymbol{S}^{\text {out }}$. Mathematically, if a solution $\boldsymbol{S}(z)$ has $\boldsymbol{S}^{\text {out }}$ as its asymptotic state and, simultaneously, its input state $\boldsymbol{S}^{\text {in }}$ belongs to $\boldsymbol{\Omega}_{\mathrm{amp}}\left(\boldsymbol{S}^{\text {out }}\right)$ then it is guaranteed that a nonlinear amplification of the SPP occurs.

The previous mechanism is clearly visualized in Fig. 5. In this figure we have represented a paradigmatic example of nonlinear SPP amplification. Our initial state is a soliplasmon state with no plasmon component at all located right at the south pole of the Earth surface, i.e., with $C_{p}^{\text {in }}=0$ and $C_{s}^{\text {in }}=E_{0}$, where $E_{0}$ is our normalization value chosen as in Secs. IV and V. Our physical parameters are the same used to find the dissipative soliplasmon pair in Fig. 4 in the previous section. This means that the gain $g$ is kept below the critical gain $g_{c}$. By solving the evolution spin equations with this initial condition we find that indeed there is a trajectory that connects the initial spin state $S^{\text {in }}=(0,0,-1)$ with the dissipative soliplasmon solution $\boldsymbol{S}_{a}$ (yellow arrow) represented in Fig. 4. The evolution process presents two different well defined phases. First, there is a process of a strong and fast amplification, which takes the initial spin towards a point of maximal amplification, represented in Fig. 5 by a white spot. Amplification is visualized in the fact that this point is located in the atmospheric shell indicating that the norm is higher than unity and thus larger than the initial one. Besides, this amplification generates plasmonic component-absent in the initial state-since we moved away from the soliton pole towards the northern hemisphere. The fact that the white spot appears visually close to the outer surface of the atmospheric shell indicates that amplification is large. The norm of the white spot is in fact larger than in any other evolution state, including the final state, thus indicating that is the point of maximal amplification. After this transient there is a slow decay from the white spot into the final stationary dissipative soliplasmon state (end point of the yellow arrow). The asymptotic value for $S(z)$ exactly coincides with the stationary spin value $S_{a}$ calculated in the previous section.

The graphic representation in the Earth-like Poincaré sphere is a faithful reflection of the behavior of the intensity evolution. The intensity can be calculated from the soliplasmon ansatz (1) since it is given by the $z$ component of the Poynting vector so that $I=E_{x} H_{y}^{*} \propto\left|E_{x}\right|^{2}$. Once the spin trajectory $\boldsymbol{S}(z)$ has been resolved, the variational parameters $C_{p}(z)$ and $C_{s}(z)$ are obtained and the soliplasmon ansatz (1) provides an explicit solution for $E_{x}(x, z)$ and thus for the intensity $I(x, z)$.

Strong and fast SPP amplification in the initial propagation stage is certainly apparent in the behavior of intensity. It occurs at typical distances of tens of microns, as visualized in Figs. 6(a) and 6(b). The peak value for the intensity corresponds to a point of maximum SPP amplification-the white spot in the sphere in Fig. 5, which for this particular configuration occurs for a propagation distance of around $13 \mu \mathrm{m}$. The nonlinear amplification of the plasmonic mode can be more neatly seen if we use the intensity of the linear SPP mode associated to the soliplasmon solution at every axial position instead of the total one. The associated SPP field is easily calculated by projecting the soliplasmon solution (1) into a normalized SPP mode_-given by the TM pair $\left[H_{p y}(x), E_{p x}(x)\right]$ 

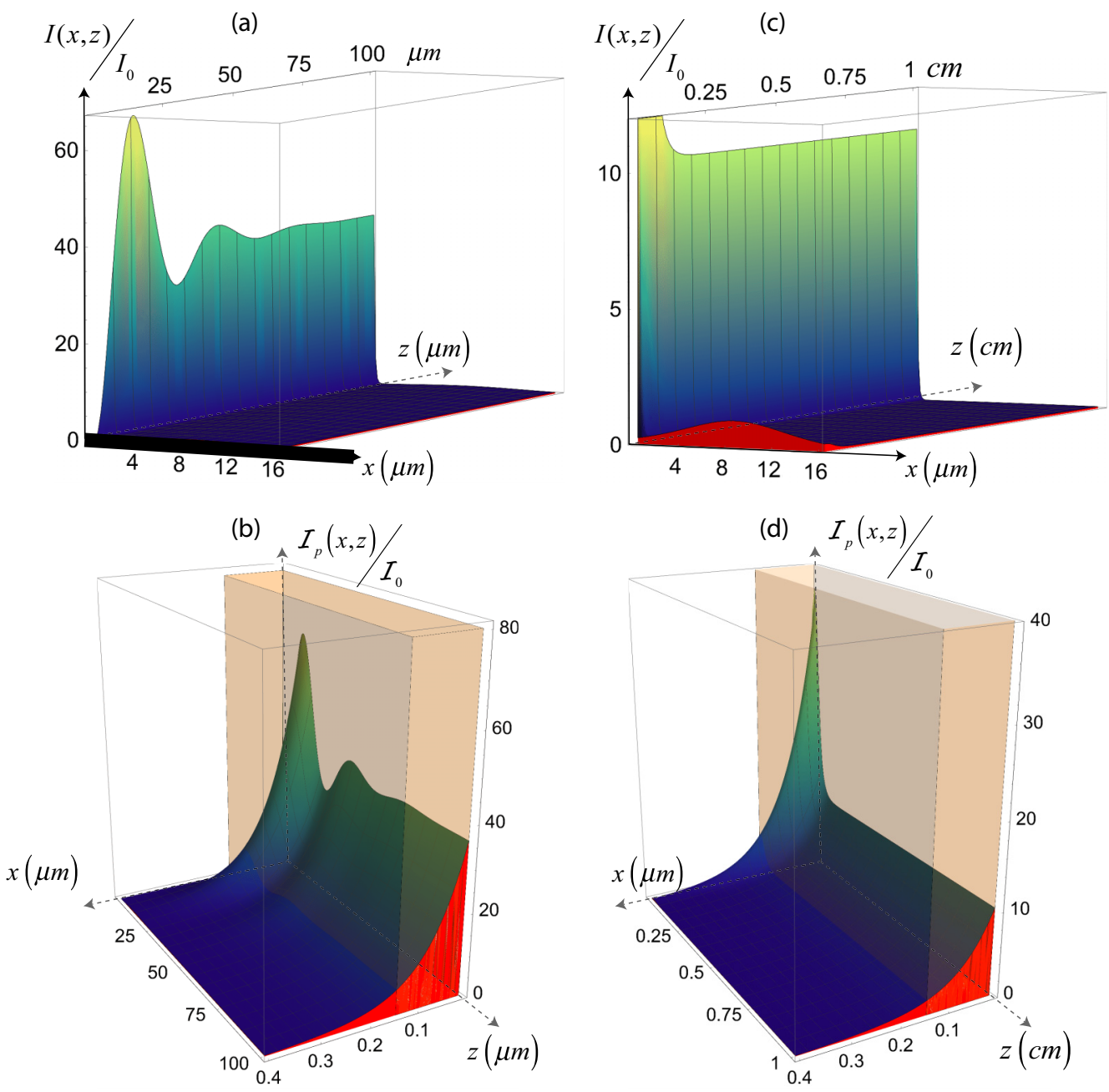

FIG. 6. Normalized intensity plots for the nonlinear SPP amplification process represented in the Earth-like Poincaré sphere of Fig. 5 for two different values of the maximum propagation length $L$. We use normalized values given by $I / I_{0}=\left|E_{x}\right|^{2} / E_{0}^{2}$, where $E_{0}$ is the normalization value referred to in the text. Physical units are used for distances. Left column graphics show the short distance behavior of the intensity for $L=20 \mu \mathrm{m}$ : (a) intensity profile of the total soliplasmon solution and (b) intensity profile of the linear SPP mode excited by the soliton. Right column shows the same evolution but for very long distances when $L=1 \mathrm{~cm}$ : (c) full soliplasmon solution and (d) linear SPP corresponding excited mode.

and corresponding to the linear waveguide constituted by the MD interface_-at every propagation step:

$$
E_{p x}^{\text {proj }}(x, z)=\left[\int d x H_{p y}^{*}(x) E_{x}(x, z)\right] E_{p x}(x) .
$$

The intensity $\mathcal{I}_{\mathrm{p}} \propto\left|E_{p x}^{\mathrm{proj}}\right|^{2}$ associated to this projection can be interpreted in fact as the intensity effectively stored into the plasmonic component of the soliplasmon mode during propagation. So it is an optimal physical magnitude to quantify the amplification-attenuation dynamics in the plasmonic waveguide. The feature of maximum SPP amplification is thus apparent in Fig. 6(b), where the normalized intensity $\mathcal{I}_{\mathrm{p}}(x, z)$ is represented up to a distance of $100 \mu \mathrm{m}$.

Asymptotic stabilization into a dissipative soliplasmon state is visualized in Figs. 6(c) and 6(d), where the propagation of the intensity is shown for a much larger distance of $L=1 \mathrm{~cm}$. The final profile in Fig. 6(c) exactly corresponds to the stationary soliplasmon solution obtained using the spin model and represented by the yellow arrow in the Earth-like Poincaré sphere (Fig. 5). The proximity to the north pole in the sphere indicates that the relative weight of the plasmonic component with respect to the solitonic one is much higher. In fact, soliton modulation is hardly visible in the intensity profile in Fig. 6(c) as soon as the SPP component stabilizes. Besides, the whole soliplasmon solution is asymptotically amplified. This feature is reflected in Fig. 5 by the fact that the end of the yellow arrow lies on the atmospheric layer. This overall amplification is smaller than for the intermediate white point, whose norm is maximum, but it goes mostly to the SPP component. The separate effect on the plasmonic component is clearly seen in Fig. 6(d), where we represent the normalized projected intensity $\mathcal{I}_{\mathrm{p}}(x, z)$ up to a distance of $1 \mathrm{~cm}$. In our example, maximal amplification of the SPP mode occurs at short distances-around $13 \mu \mathrm{m}$-but asymptotic amplification is achieved after around $200 \mu \mathrm{m}$. From this point on, infinitely long stationary propagation occurs provided the system conditions are preserved. 


\section{GAIN-LOSS BALANCE AND ENERGY FLUX}

The plasmonic intensity $\mathcal{I}_{\mathrm{p}}$ introduced in the previous section can be also used to understand the gain-loss dynamics of the soliplasmon system. Its spatial integral $I_{p}(z)=$ $\int d x \mathcal{I}_{p}(x, z)$ can be systematically evaluated at every axial distance once the variational parameters $C_{p}(z)$ and $C_{s}(z)$ are determined. The complete knowledge of the function $I_{p}(z)$ permits one to introduce the concept of effective plasmonic gain function $g_{p}^{\text {eff }}(z)$ in the following way:

$$
\frac{d I_{p}(z)}{d z}=2\left(g_{p}^{\mathrm{eff}}(z)-l\right) I_{p}(z)
$$

This form permits one to understand the soliplasmon stabilization dynamics in an analogous way as the onset of laser oscillations in an idealized laser cavity with losses. Our equation for $I_{p}(25)$ is analogous to the photon rate equation for a lossy laser cavity in which the threshold gain - the minimum gain compensating all cavity losses-is given here by the SPP loss coefficient $l$ [53]. As in the laser cavity, also a nonlinear dissipative system, the steady state is only achieved when gain balances loss completely after a nontrivial transient. We can see in Fig. 7(a) how the stabilization of the dissipative soliplasmon solution can be neatly interpreted in terms of the balance between the effective plasmonic gain $g_{p}^{\text {eff }}$-calculated using Eq. (25) — and the plasmonic loss. Indeed, the perfect gain-loss balance condition for the plasmonic component is asymptotically fulfilled

$$
g_{p}^{\text {eff }}=l .
$$

In fact, the gain-balance condition (26) defines the onset of the dissipative soliplasmon resonance. It is remarkable that this condition can be achieved despite the nominal linear gain in the Kerr medium $g$ being much smaller than $l$.

In order to understand the physical origin of the effective plasmonic distributed gain, we need to focus on the energy flux dynamics within the soliplasmon structure. If we consider the SPP subsystem as the one defined by the domain $\Omega_{\mathrm{MD}}$ $(x \leqslant d)$-containing most of the SPP amplitude, Eq. (25) can be understood as the continuity equation for the energy stored in the plasmonic subsystem. The second term in Eq. (25) accounts for the rate of energy absorbed by an electromagnetic "sink" present in the SPP subsystem, whose origin is nothing but the losses at the metal layer. The first term is thus naturally interpreted as the flux of electromagnetic energy traversing the boundary of our selected domain $\Omega_{\mathrm{MD}}$, which is given by the transverse flux at $x=d$. Thus this transverse flux $\Phi_{t}$ has to be proportional to the $x$ component of the Poynting vector at this point: $\Phi_{t}(z) \propto S_{x}(d, z)$. In the absence of gain $(g=0)$ this situation is qualitatively reflected in Fig. 1(a). Plasmonic losses act as an electromagnetic energy sink partially compensated by the flux of energy arising from the soliton. However, this soliton-to-plasmon energy flux is not enough to compensate plasmonic losses so that the plasmonic component continuously decays and eventually disappears. This qualitative analysis is confirmed by the numerical simulations of soliplasmon propagation using the full-vector nonlinear Maxwell's equations in Ref. [34].

As we will see next, the nontrivial features of the energy flux dynamics in our previous numerical simulation are also captured by our soliplasmon model in a very appealing way. By construction, the energy stored in the plasmonic subsystem can be easily evaluated using the soliplasmon model since, as a first approximation, $I_{p}(z)=K\left|C_{p}(z)\right|^{2}$, where $K$ is a proportionality constant. By writing the variational components in Eqs. (7) as $C_{p, s}(z)=\left|C_{p, s}(z)\right| e^{i \phi_{p, s}(z)}$ and separating real and imaginary parts, we can obtain an equation for $d\left|C_{p}\right| / d z$. Since $d I_{p} / d z=2 K\left|C_{p}\right| d\left|C_{p}\right| / d z$ we obtain immediately

$$
\frac{d I_{p}(z)}{d z}=2\left(K q \frac{\left|C_{p}(z)\right|}{\left|C_{s}(z)\right|} \sin \phi_{s p}(z)-l\right) I_{p}(z) .
$$

By comparing Eq. (25) to Eq. (27) and according to our previous arguments regarding the transverse energy flux, it becomes clear that the following relations are fulfilled:

$$
\Phi_{t}(z) \propto g_{p}^{\text {eff }}(z)=K q \frac{\left|C_{p}(z)\right|}{\left|C_{s}(z)\right|} \sin \phi_{s p}(z) .
$$

This equation permits one to understand the structure of the electromagnetic energy flux in a stationary dissipative soliplasmon. For a stationary dissipative soliplasmon the absolute values $\left|C_{p}\right|$ as $\left|C_{s}\right|$ are $z$ independent (consequently,
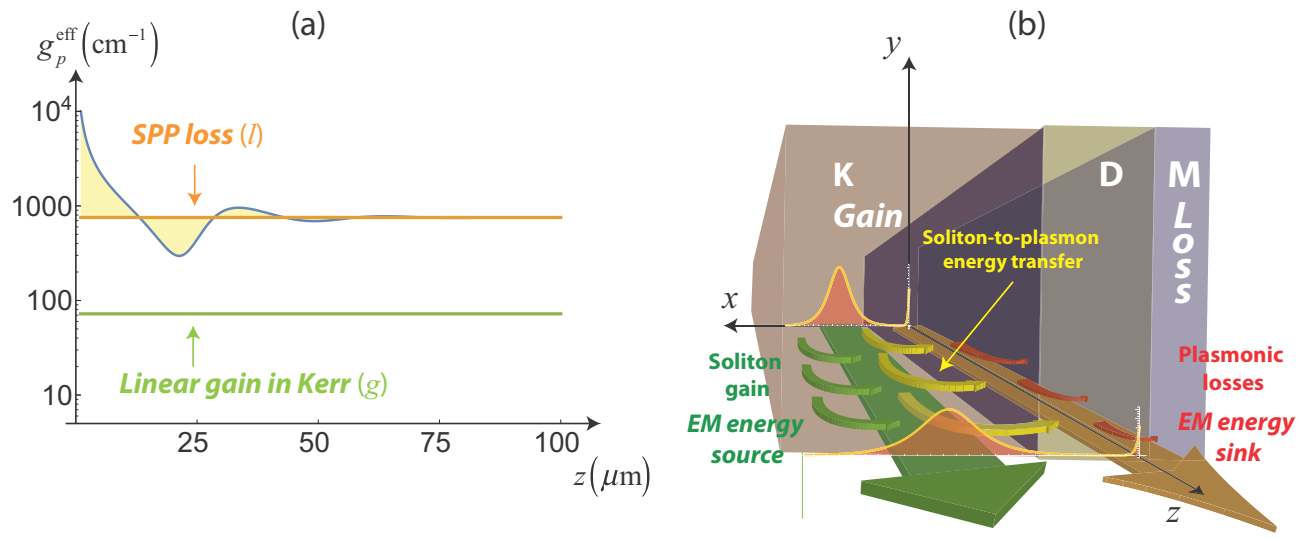

FIG. 7. (a) Effective gain as a function of distance showing perfect asymptotic loss compensation-we restore physical units ( $\left.\mathrm{cm}^{-1}\right)$ to gain and loss coefficients and take the same configuration and parameters as in Secs. VI and VII. (b) Graphical representation of the typical energy flux configuration for a stationary dissipative soliplasmon with gain in the Kerr medium compensating for SPP losses. 
so is $q$ ) as well as the relative phase $\phi_{s p}$. Therefore, by comparing Eq. (28) and the balance condition (26) we immediately recognize that, once the modulus $\left|C_{s}\right|$ is fixed by the golden constraint (and thus also $\left|C_{p}\right|$ ), the value of the relative phase cannot be arbitrary. In terms of the energy flux, $\phi_{s p}$ has to adjust its value in order to guarantee an energy flux that balances plasmon losses completely. For our particular solution in Fig. 4, for example, $\phi_{s p} \approx \pi / 8$. Put differently, in a stationary dissipative soliplasmon the relative phase $\phi_{s p}$ determines the exact electromagnetic energy flux flowing from the soliton to the SPP compensating plasmon losses in an exact manner. The equilibrium is achieved since the electromagnetic gain in the Kerr medium is perfectly in balance with loss in the metal through the soliton-to-plasmon energy flux, as graphically depicted in Fig. 7(b).

It is remarkable how the soliplasmon model explains why the existence of a nonzero flux balance is not achievable when there is no gain in the Kerr medium. When $g=0$, the golden constraint as written in Eq. (23) tells us that the product $q^{\prime}\left(C_{s}\right) \bar{q}^{\prime}\left(C_{s}\right)$ has to vanish, which implies that the only solution is the trivial one $\left|C_{s}\right|=0$. The soliton component disappears in the soliplasmon model equations (7) leaving only a decaying plasmonic component that asymptotically vanishes: $\left|C_{p}\right| \rightarrow 0$. Thus no nontrivial dissipative solutions can exist.

A different situation occurs in the conservative case, when $l=g=0$. As mentioned in Sec. V, the golden constraint disappears. In addition and according to the balance equation (26), since there is no loss to compensate the effective plasmonic gain is zero, Thus the electromagnetic transverse flux $\Phi_{t}$ has to vanish, which, according to Eq. (28), implies that the relative soliplasmon phase can only be either 0 or $\pi$. This is exactly the situation describing the conservative soliplasmons depicted in Fig. 2. Inasmuch as there is no golden constraint we have the two families of 0 and $\pi$ soliplasmon solutions parametrized by a continuous range of values of $\left|C_{s}\right|$.

\section{CONCLUSIONS}

In this work we have established a gain-loss generalization of the mechanism for the excitation of lossy plasmonic modes using spatial solitons previously reported in $[33,34]$. The key point is that this excitation mechanism occurs in the neighborhood of a soliplasmon resonance. In this generalization we define a noteworthy strategy to introduce gain in the system. Linear gain $g$ is introduced in the region where the nonlinear Kerr medium is located [see Fig. 1(b)]. The strategy of including gain in the Kerr medium is suggested by the previous mechanism of nonlinear excitation of SPPs, in which the existence of a soliplasmon resonance plays a crucial role. The nearly resonant state formed by the SPP and the soliton-the soliplasmon resonance-exhibits the phenomenon of anticrossing [33], thus indicating the possibility of adiabatically (or diabatically if loss and gain are introduced) making transitions between the two modes [54]. In our case, numerical simulations of full-vector nonlinear equations have shown that the soliton is able to excite its SPP partner in the presence of plasmonic losses in a relatively efficient manner, increasing the SPP propagation length [34]. For this reason, we expect that adding a moderate gain in the Kerr medium can considerably enhance this effect.
Indeed, we have proven that by adding a small imaginary part to the soliton propagation constant $\mu_{s}=\mu_{s}^{\prime}-i k_{0} g$ it is possible to compensate plasmonic losses completely. With gain coefficients $g$ significantly smaller than the plasmonic loss $l$ it is possible to excite a soliplasmon resonance in a way that the imaginary part of its propagation constant $\mu$ becomes exactly zero, so that the propagation length associated to the SPP component of the coupled system becomes infinite.

The key point of this nonlinear mechanism of plasmonic amplification is, however, not only the existence of a resonant interaction between the SPP and the soliton but that this interaction is dictated by an asymmetric and nonlinear evanescent coupling. It is the fact that the two different coupling coefficients depend on the soliton amplitude as $q, \bar{q} \propto$ $\exp \left(-K\left|C_{s}\right| a\right)$, which makes the existence of stationary dissipative soliplasmons-qualitatively and quantitativelydifferent from what one would obtain by assuming standard linear couplings (i.e., independent of $\left|C_{S}\right|$ ). In particular, the presence of a nonlinear evanescent coupling between the SPP and the soliton predicts the existence of two dissipative solutions always, provided gain $g$ remains below a critical maximum value $g_{c}$. As a corollary, our model predicts that nonlinear plasmonic amplification is critical $\left(g \leqslant g_{c}\right)$ and asymmetric $(l \gg g)$. The same analysis using a linear coupling instead leads to a completely different prediction (see Fig. 3.) The nonlinear evanescent coupling is a direct consequence of the soliplasmon ansatz used to obtain the variational equations leading to the soliplasmon model (7), as rigorously demonstrated from nonlinear Maxwell's equations in Ref. [47]. Asymmetry between the plasmon-to-soliton coupling $\bar{q}$ and the soliton-to-plasmon coupling $q$ in realistic configurations $(\bar{q} \ll q)$ confers also peculiar properties to the soliplasmon model and, thus, to the nonlinear mechanism of plasmonic amplification associated to it.

The dissipative soliplasmon model presented here can be applied to other solutions than a SPP and a spatial soliton. It can be applied to a more general plasmonic mode of a 1D or $2 \mathrm{D}$ generic plasmonic waveguide coupled to a spatially separated nonlinear dielectric mode associated to an intricate dielectric/Kerr waveguide [47]. For 1D structures, for example, the plasmonic mode can be a LRSPP and the dielectric mode can represent the nonlinear mode of a dielectric/Kerr/dielectric waveguide. The qualitative features of the mechanism of nonlinear plasmonic amplification mediated by dissipative soliplasmon resonances here presented apply then to a more general set of plasmonic structures. In this direction, the dissipative soliplasmon model (7), or, equivalently, its spin model version (11), describes a general behavior shared by more gain-assisted nonlinear plasmonic guiding structures. We focused here on the features associated to plasmonic amplification since compensation of plasmonic losses has been the main motivation of this work. However, the particular nature of the soliplasmon model, with its peculiar asymmetric and nonlinear evanescent coupling, makes this model certainly much richer, as previous analysis for the particular case of symmetric coupling shows [49]. In this sense, the use of known techniques developed to analyze dissipative solitons in nonlinear systems [52] can be applied to describe properly the nature of all potential solutions of the soliplasmon model beyond those found in the present work. 
Last but not the least, the soliplasmon model (7) presents interesting connections to parity-time (PT) symmetric optical systems $[55,56]$. It can be related to the discrete model describing the so-called nonlinear PT-invariant dimers, represented physically by two linearly coupled active and passive nonlinear waveguides with balanced gain and loss [57-60]. Despite the non-Hermiticity of the Hamiltonian these PTsymmetric systems admit stationary dissipative soliton solutions, in which loss is fully balanced by gain. In our model the coupling is both asymmetric and nonlinear and, nevertheless, stationary dissipative soliplasmons are equally found with unbalanced gain and loss. Interestingly, in other structures with unbalanced gain and loss, mathematically represented by coupled Ginzburg-Landau equations but with linear and symmetric coupling, it is possible to find also stable dissipative solitons [61-63]. In this context, the dissipative soliplasmon appears as a peculiar form of a dissipative soliton, whose existence and properties rely on the asymmetric and the nonlinear evanescent character of the plasmon-soliton coupling near resonance. In this article we have used some of the particular properties of the dissipative soliplasmon model aiming at optimizing plasmonic amplification. However, many other features of the model are still unexplored. Since nonlinear plasmonic amplification occurs due to the mediation of the dissipative soliplasmon quasiparticle, it is of great interest for other potential applications to deepen in the distinguishing properties of this type of solution.

\section{ACKNOWLEDGMENTS}

This work was supported by the MINECO (Government of Spain) under Grants No. TEC2010-15327, No. TEC201350416-EXP, and No. TEC2014-53727-C2-1-R.

\section{APPENDIX A: VARIATIONAL EQUATIONS}

The variational equations describing the interaction of a linear surface plasmon polariton (SPP) and a spatial soliton propagating in parallel to a metal/dielectric/Kerr (MDK) double interface were demonstrated in Ref. [47] (see Fig. 1). The linear behavior of this system is described by a permittivity function given by

$$
\varepsilon_{L}(x)= \begin{cases}\varepsilon_{p}(x), & x \leqslant d, \\ \varepsilon_{\mathrm{K}}, & x>d\end{cases}
$$

where $d$ is the thickness of the dielectric layer, $\varepsilon_{K}$ is the dielectric constant of the Kerr medium, and $\varepsilon_{p}(x)$ is the profile of the permittivity that defines the MD interface where the SPP propagates, i.e.,

$$
\varepsilon_{p}(x) \equiv \begin{cases}\varepsilon_{m} & \text { if } x \leqslant 0 \\ \varepsilon_{d} & \text { if } x>0\end{cases}
$$

$\varepsilon_{m}$ and $\varepsilon_{d}$ being the dielectric constants of the metal and the dielectric, respectively. In a first approach, the metal is considered to be ideal so that $\operatorname{Im}\left(\varepsilon_{m}\right)=0$.

The resonant interaction between the spatial soliton and the SPP is well described by a variational $T M$ solution for the electric field $\mathbf{E}(x, z)=\left(E_{x}, 0, E_{z}\right)$ of the form

$$
E_{x}(x, z)=C_{p}(z) e_{p}(x)+C_{s}(z) \operatorname{sech}\left[\sqrt{\frac{\gamma}{2}}\left|C_{s}(z)\right|(x-a)\right],
$$

where

$$
e_{p}(x)=\varepsilon_{p}^{-1}(x)\left\{\begin{array}{ll}
e^{\kappa_{m} x}, & x \leqslant 0 \\
e^{-\kappa_{d} x}, & x>0
\end{array} \equiv \varepsilon_{p}^{-1}(x) f_{p}(x)\right.
$$

represents the linear SPP field, $k_{m}$ and $k_{d}$ being the inverse penetration lengths in the metal and dielectric, respectively. These quantities are explicit functions of the dielectric constants of the metal and of the dielectric [1,2]:

$$
\begin{gathered}
\kappa_{m}=-k_{0} \varepsilon_{m}\left[-\left(\varepsilon_{m}+\varepsilon_{d}\right)\right]^{-1 / 2}, \\
\kappa_{d}=k_{0} \varepsilon_{d}\left[-\left(\varepsilon_{m}+\varepsilon_{d}\right)\right]^{-1 / 2},
\end{gathered}
$$

with $k_{0}$ being the vacuum wave number.

The amplitude of the SPP, $C_{p}(z)$, is the first complex variational parameter. The second term in Eq. (A3) represents a soliton located at a distance $a$ from the MD interface and it has the standard form of a sech function with an amplitude $C_{s}(z)$, which constitutes the second variational parameter of the model. The nonlinear coefficient $\gamma$ in the soliton functional expression is given by $\gamma=(3 / 4) \varepsilon_{0} c n_{2}$, where $n_{2}$ is the ordinary nonlinear index of the Kerr medium. As one can see, all the nonvariational parameters of the ansatz (A3) are fixed. The soliton position $a$ is an input of the model as well as the rest of the nonvariational parameters, which are given in terms of the physical constants defining the MDK structure.

In this variational approach the axial component $E_{z}$ can be obtained approximately using a transversality constraint once $E_{x}$ is determined. The variational equations for $C_{p}$ and $C_{s}$ are obtained under the following assumptions: (i) propagation is quasistationary (i.e., phases of variational parameters are assumed to change faster than their modulus), (ii) propagation is paraxial and preserves the quasitransverse condition $\left(\left|E_{z}\right| \ll\left|E_{x}\right|\right)$, and (iii) the SPP-soliton coupling is small [i.e., overlapping of SPP and soliton amplitudes in Eq. (A3) is small]. The variational parameters $C_{p}(z)$ and $C_{s}(z)$ define then the solution completely through the following nonlinear model of coupled oscillators:

$$
\begin{aligned}
-i \frac{d C_{p}}{d z} & =\mu_{p} C_{p}+q\left(C_{s}\right) C_{s}, \\
-i \frac{d C_{s}}{d z} & =\mu_{s}\left(C_{s}\right) C_{s}+\bar{q}\left(C_{s}\right) C_{p},
\end{aligned}
$$

where

$$
\begin{aligned}
& \mu_{p} \equiv \frac{\left(\beta_{p}^{2}-k_{0}^{2} \varepsilon_{\mathrm{K}}\right)}{2 k_{0} \varepsilon_{\mathrm{K}}^{1 / 2}}, \\
& \mu_{s}\left(C_{s}\right) \equiv \frac{\left(\beta_{s}^{2}-k_{0}^{2} \varepsilon_{\mathrm{K}}\right)}{2 k_{0} n_{\mathrm{K}}}=\frac{k_{0} \gamma}{4 \varepsilon_{\mathrm{K}}^{1 / 2}}\left|C_{s}\right|^{2}
\end{aligned}
$$

are the paraxial propagation constants of the plasmon and soliton, respectively. As it is well known, the plasmon nonparaxial propagation constant $\beta_{p}$ is explicitly given in terms of the dielectric and metal dielectric 
constants $[1,2]$ :

$$
\beta_{p}=\sqrt{\frac{\varepsilon_{m} \varepsilon_{d}}{\varepsilon_{m}+\varepsilon_{d}}},
$$

whereas the paraxial soliton propagation $\mu_{s}$ in Eqs. (A7), as expected, presents a quadratic nonlinear dependence on the soliton variational parameter $C_{s}$, since

$$
\beta_{s}=k_{0}\left(\varepsilon_{\mathrm{K}}+\frac{\gamma}{2}\left|C_{s}\right|^{2}\right)^{1 / 2} .
$$

Remarkably, this is not the only nonlinearity of this coupled oscillator model. The variational calculation in Ref. [47] predicts that the coupling coefficients in Eqs. (A6) exhibit also an implicit nonlinear dependence on the soliton variational parameter $C_{s}$ through the overlapping integrals over the MD and soliton domains $\Omega_{\mathrm{MD}}(x \leqslant d)$ and $\Omega_{s}(x>d)$ :

$$
\begin{aligned}
q\left(C_{s}\right) \equiv & \frac{k_{0}}{2 \varepsilon_{K}^{1 / 2} N_{p}} \int_{\Omega_{\mathrm{MD}}} d x f_{p}(x) \triangle \varepsilon_{s}(x) \operatorname{sech}\left[\sqrt{\frac{\gamma}{2}}\left|C_{s}\right|(x-a)\right], \\
\bar{q}\left(C_{s}\right) \equiv & \frac{k_{0}}{2 \varepsilon_{K}^{1 / 2} N_{s}} \int_{\Omega_{s}} d x f_{p}(x) \triangle \varepsilon_{p}(x) \operatorname{sech}\left[\sqrt{\frac{\gamma}{2}}\left|C_{s}\right|(x-a)\right] \\
& +\bar{q}_{V}\left(C_{s}\right),
\end{aligned}
$$

where $\Delta \varepsilon_{s}=\varepsilon_{L}-\varepsilon_{s}$ and $\Delta \varepsilon_{p}=\varepsilon_{L}-\varepsilon_{p}$ are the local permittivity functions, which are also completely determined by the dielectric constants of the metal, of the linear dielectric, and of the Kerr medium in the following way:

$$
\Delta \varepsilon_{p}(x)= \begin{cases}0, & x \leqslant d, \\ \varepsilon_{\mathrm{K}}-\varepsilon_{d}, & x>d\end{cases}
$$

and

$$
\Delta \varepsilon_{s}(x)= \begin{cases}\varepsilon_{m}-\varepsilon_{\mathrm{K}}, & x \leqslant 0, \\ \varepsilon_{d}-\varepsilon_{\mathrm{K}}, & 0<x \leqslant d, \\ 0, & x>d,\end{cases}
$$

$d$ being the width of the linear dielectric slab (see Fig. 1). The term $\bar{q}_{V}$ is the contribution to the plasmon-to-soliton coupling of the vector term associated to the gradient of the SPP dielectric function $\varepsilon_{p}^{-1} \nabla \varepsilon_{p}$. There is no such contribution for the soliton-to-plasmon coupling $q$ because we consider the soliton to fulfill a scalar equation. Note that, according to their definitions, the local permittivity functions $\Delta \varepsilon_{p}$ and $\Delta \varepsilon_{s}$ are only nonzero in the soliton $\Omega_{s}$ and $\Omega_{\mathrm{MD}}$ domains, respectively. This property justifies the integration domains in Eqs. (A9). On the other hand, the parameters $N_{p}$ and $N_{s}$ are normalization constants, which are given in terms of the plasmon and soliton inverse penetration lengths as

$$
\begin{aligned}
& N_{p}=\int d x f_{p}^{2}(x) \approx \frac{1}{2}\left(\kappa_{d}^{-1}+\kappa_{m}^{-1}\right), \\
& N_{s}=\int d x \operatorname{sech}^{2}\left[\sqrt{\frac{\gamma}{2}}\left|C_{s}\right|(x-a)\right] \approx 2 \kappa_{s}^{-1} .
\end{aligned}
$$

Note that since the soliton penetration length also depends on the shape of the soliton profile, there is an extra dependence on the soliton variational parameter given by $\kappa_{s}=(\gamma / 2)^{1 / 2} k_{0}\left|C_{S}\right|$. This fact along with the expressions for the coupling coefficients (A9) clearly point out both the asymmetry and the nontrivial nonlinear nature of the resonant soliplasmon coupling.

The variational model given by the nonlinear coupled oscillator system (A6) is a model with no free parameters. All coefficients entering these equations are given in the last instance in terms of the optical coefficients that characterize the MDK structure-i.e., $\varepsilon_{m}, \varepsilon_{d}, \varepsilon_{K}$, the width $d$, and the nonlinear index $n_{2}$-along with the soliton position $a$. In this sense, within the regime of validity of the approximations used to obtain it, the variational model possesses the same predictive power as the original Maxwell's equations from which it was derived.

The inclusion of metal losses $\left[\operatorname{Im}\left(\varepsilon_{m}\right) \neq 0\right]$ does not change the equations for the SPP. They remain valid assuming now that $\varepsilon_{m}$ is complex [1]. For this reason the structure of all the previous equations does not change. However, now the plasmon function $f_{p}$ is complex since both $\kappa_{m}$ and $\kappa_{d}$ are, according to Eq. (A5). The SPP propagation constant (A8) becomes complex as well:

$$
\beta_{p}^{2}=\beta_{p}^{\prime 2}+i \beta_{p}^{\prime 2}
$$

Consequently, and according to (A7), the paraxial propagation constant becomes also complex: $\mu_{p}=\mu_{p}^{\prime}+i \mu_{p}^{\prime \prime}$. The same occurs to the coupling coefficients in (A9), which now can be written explicitly separating their real and imaginary parts as

$$
\begin{aligned}
& q=q_{m}^{\prime}+i \delta q_{m}^{\prime \prime}, \\
& \bar{q}=\bar{q}_{m}^{\prime}+i \delta \bar{q}_{m}^{\prime \prime} .
\end{aligned}
$$

We use the subindex $m$ to indicate that the origin of these terms is the existence of metal losses, so that $\delta q_{m}^{\prime \prime}, \delta \bar{q}_{m}^{\prime \prime} \rightarrow 0$ when $\operatorname{Im}\left(\varepsilon_{m}\right) \rightarrow 0$.

\section{APPENDIX B: EARTH-LIKE POINCARÉ SPHERE}

The Poincaré sphere (or Bloch sphere) is a standard representation of a density matrix, which is by construction Hermitian and positive definite $(\operatorname{det} \rho \geqslant 0)$ [64]. The general construction followed in Sec. III to write arbitrary Hermitian matrices in the $\left\{\tau_{0}, \tau\right\}$ basis determines that a generic density matrix $\rho$ is represented by a four-dimensional vector $\left(S_{0}, S\right)$ verifying the inequality $|\boldsymbol{S}| \leqslant S_{0}$. This inequality defines a sphere of radius $S_{0}$ (the Poincaré or Bloch sphere) in the 3D space of spin components. A pure state represented by a density matrix $\rho=|C\rangle\langle C|$ is the only type of state that fulfills the equality $|\boldsymbol{S}|=S_{0}$ having the explicit representation in terms of the components of the complex vector $C$ given in Eq. (13). Pure states correspond then to vectors lying on the surface of the Poincaré sphere. Nonpure states are represented by spin vectors inside the Poincaré sphere.

When we are considering the conservative case, in which we set $\sigma=0$ in the spin model evolution equation (11), $|\boldsymbol{S}|$ is conserved and, therefore, all the allowed trajectories of the spin $\boldsymbol{S}(z)$ occur on the surface of the Poincaré sphere. In the linear case, the spin vector $S(z)$ experiments a precession around the constant effective magnetic field $\boldsymbol{\Omega}$. In the nonlinear case, since $\boldsymbol{\Omega}$ depends on $\boldsymbol{S}$, the magnetic field can also evolve in $z$. However, no matter the evolution is linear or nonlinear, the conservative character of the dynamics preserves the norm of the spin and, therefore, the condition that $S$ has to move on the surface of the Poincaré sphere. 
When the spin system is nonconservative, we are in the general case described by the spin evolution equations (11) with $\sigma \neq 0$. Now, the first equation for $d S_{0} / d z$ clearly indicates that the evolution of the spin vector does not preserve its norm. As a consequence, evolution can take the spin vector out of the surface of the Poincaré sphere. In the general case it is possible that $d S_{0} / d z$ is negative or positive so we can find situations in which $|\boldsymbol{S}|<S_{0}$ or $|\boldsymbol{S}|>S_{0}$. Consequently, in the nonconservative case we expect that the trajectories of the spin vectors move all over the space, inside and outside the Poincaré sphere.

However, the expression that we found for the zero component of a stationary dissipative soliplasmon (22) tells us that we can find solutions with an infinitely large value of $S_{0}$ (when $n_{3} \rightarrow 1$ ). The existence domain of $S_{0}$ is then the whole positive real axis $[0, \infty[$. Since the visualization of spin vectors with large norm can be difficult using the standard Poincaré sphere, we introduce a modified version of this representation. Basically, we introduce a new function that maps the infinite $S_{0}$ domain into the finite domain $[0, \varphi]$, where $\varphi$ is the golden ratio. The choice of $\varphi$ is certainly arbitrary. It is chosen because it provides a good proportion between the spheres radii for an equilibrated visualization of the inner and outer sphere:

$$
\begin{gathered}
\bar{S}_{0}(z)=\frac{2 \varphi}{\pi} \arctan \left[\frac{S_{0}(z)}{\widetilde{S}} \tan \left(\frac{\pi}{2 \varphi}\right)\right], \\
\bar{S}(z)=\bar{S}_{0}(z) \frac{\boldsymbol{S}(z)}{|\boldsymbol{S}(z)|},
\end{gathered}
$$

where $\widetilde{S}$ is some reference value. The previous mapping has the following properties:

$$
\begin{array}{ll}
\bar{S}_{0} \rightarrow 0 & \text { when } S_{0} \rightarrow 0 \\
\bar{S}_{0} \rightarrow 1 & \text { when } S_{0} \rightarrow \widetilde{S} \\
\bar{S}_{0} \rightarrow \varphi & \text { when } S_{0} \rightarrow \infty
\end{array}
$$

In fact, the particular form of the mapping (B1) is to some extent arbitrary since another mapping function could be used provided it satisfied the previous requirements together with the properties of analyticity and monotonicity in the $[0, \infty[$ domain. The advantage of such a mapping is that now all spin vectors generated by the evolution described by the nonconservative spin equations (11) fit always in an extended Poincaré sphere of radius $R=\varphi$. Since $\bar{S}_{0}$ is a monotonically increasing function it is also true that

$$
\begin{aligned}
& |S|<\widetilde{S} \Rightarrow 0 \leqslant \bar{S}_{0}<1, \\
& |S|=\widetilde{S} \Rightarrow \bar{S}_{0}=1, \\
& |S|>\widetilde{S} \Rightarrow 1<\bar{S}_{0} \leqslant \varphi .
\end{aligned}
$$

According to these properties, the function (B1) maps the inner part and the surface of a Poincaré sphere (of radius $R=\widetilde{S}$ ) into a normalized to unit Poincaré sphere. However, the infinite outer part is mapped into a finite shell comprised between the unit sphere and an outer sphere of radius equal to the golden ratio $\varphi$.

For example, if we chose to analyze the spin motion in a conservative regime in which $\sigma=0$ and thus $|\boldsymbol{S}|=S_{0}^{c}$ is conserved, we would select $\widetilde{S}=S_{0}^{c}$ and therefore all the motion would happen on the surface of the unit sphere. If we now switched on the nonconservative terms in Eq. (11) by letting $\sigma \neq 0$, the motion would cease to be on the surface and either it would "take off" into the outer shell (if $d S_{0} / d z>0$ ) or it would "dig into" the inner one $\left(d S_{0} / d z<0\right)$. In this framework, the outer shell represents the set of spin states with overall gain (larger norm) with respect to their counterparts with $\sigma=0$. Equivalently, the inner shell constitutes the set of states with overall loss (smaller norm) with respect to the same spin states with $\sigma=0$. The physical picture of this extended version of the standard Poincare sphere is similar to an Earth-like system, in which conservative motion occurs on the Earth surface, lossy evolution takes place under the Earth surface, and evolution with gain materializes into the Earth atmosphere. For this reason, we refer to this form of representing the solutions of the nonconservative spin model (11) as the Earth-like Poincaré sphere.

\section{APPENDIX C: PROPAGATION CONSTANT OF A STATIONARY DISSIPATIVE SOLIPLASMON}

In the framework of our spin model, a fully determined solution of a stationary dissipative soliplasmon is given by the spin equations (21) and (22). Apparently, however, the information about the propagation constant of the original stationary state $C=\left(C_{p}, C_{s}\right)^{\top}$ seems to be lost in the process of defining the equivalent spin model. Indeed, this essential parameter does not appear explicitly in the dynamical spin equations (11) nor in the solutions for stationary spin states (15). On the other hand, the general form of the spin vector $S$ in terms of the components of the $C$ vector (13) only determines the modulus $\left|C_{p}\right|,\left|C_{s}\right|$ of the plasmon and soliton components as well as their relative phase $\phi_{s p}=\phi_{s}-\phi_{p}$ but not the individual phase of each component. In this way, given the spin vector of a stationary solution, we can know the stationary state $C$ up to a global phase, which in principle could depend on $z$ in an arbitrary form:

$$
C(z)=e^{i \phi_{p}(z)}\left[\begin{array}{c}
\left|C_{p}(0)\right| \\
\left|C_{s}(0)\right| e^{i \phi_{s p}(0)}
\end{array}\right]=e^{i \phi_{p}(z)} \widetilde{C},
$$

where $\widetilde{C}$ is the $2 \mathrm{D}$ complex vector that can be univocally constructed from the values of the stationary spin solution.

Despite this fact, the propagation constant of the stationary dissipative soliplasmon is also univocally defined. According to our process of constructing our dissipative nonlinear solution, once we determine-if extant-the specific value of $\left|C_{S}\right|$ that satisfies the golden constraint, all components of the original nonlinear Hamiltonian $H(9)$ are fixed. This is so because $q, \bar{q}$, and $\mu_{s}$ are functions of $\left|C_{s}\right|$, whereas $\mu_{p}$ is a constant. According to the original Hamiltonian equation for the vector $C(9)$, the stationary solution $(\mathrm{C} 1)$ would fulfill this equation in the following way:

$$
\frac{d \phi_{p}}{d z} \widetilde{C}=H \widetilde{C},
$$

where the matrix $H$ and the vector $\widetilde{C}$ are both independent of $z$ and fully determined by the stationary spin solution. The solution of the equation above is thus

$$
\phi_{p}(z)=\mu z
$$


where

$$
\mu=\frac{\widetilde{C}^{\dagger} H \widetilde{C}}{\widetilde{C}^{\dagger} \widetilde{C}}=\frac{\operatorname{Tr}(\widetilde{\rho} H)}{\operatorname{Tr}(\widetilde{\rho})},
$$

in which $\widetilde{\rho}=\widetilde{C} \widetilde{C}^{\dagger}$ is the density matrix associated to the stationary spin solution. We can rewrite the previous equation in terms of the real four-dimensional vectors $\Omega, \sigma$, and $S$ following the procedure in Sec. III:

$$
\begin{aligned}
\mu & =[\operatorname{Tr}(\Pi \widetilde{\rho})+i \operatorname{Tr}(\Sigma \widetilde{\rho})] / \operatorname{Tr}(\widetilde{\rho}) \\
& =\frac{1}{2}\left(\Omega_{0}+\boldsymbol{\Omega} \cdot \boldsymbol{n}\right)+i \frac{1}{2}\left(\sigma_{0}+\boldsymbol{\sigma} \cdot \boldsymbol{n}\right) .
\end{aligned}
$$

However, for stationary spin solutions the imaginary part vanishes. The reason is that the imaginary part of $\mu$ is proportional to $d S_{0} / d z$ since, according to the first of the spin equations (11), we have

$$
\operatorname{Im}(\mu)=\frac{1}{2}\left(\sigma_{0}+\boldsymbol{\sigma} \cdot \boldsymbol{n}\right)=-\frac{1}{S_{0}} \frac{d S_{0}}{d z} .
$$

Inasmuch as stationary solutions have invariant norm they fulfill the condition $d S_{0} / d z=0$ and, therefore, $\operatorname{Im}(\mu) \sim$ $d S_{0} / d z=0$.

In summary, we have seen that the propagation constant of a stationary dissipative soliplasmon is real and it is univocally defined by the components of the stationary spin solution associated to it as

$$
\mu=\frac{1}{2}\left[\Omega_{0}+\boldsymbol{\Omega} \cdot \boldsymbol{n}\right] .
$$

[1] S. A. Maier, Plasmonics: Fundamentals and Applications (Springer, New York, 2007).

[2] J. M. Pitarke, V. M. Silkin, E. V. Chulkov, and P. M. Echenique, Rep. Prog. Phys. 70, 1 (2007).

[3] V. M. Agranovich, V. S. Babichenko, and V. Y. Chernyak, JETP Lett. 32, 512 (1980).

[4] D. Mihalache, R. G. Nazmitdinov, and V. K. Fedyanin, Phys. Scr. 29, 269 (1984).

[5] G. I. Stegeman, C. T. Seaton, J. Ariyasu, R. F. Wallis, and A. A. Maradudin, J. Appl. Phys. 58, 2453 (1985).

[6] J. Ariyasu, C. T. Seaton, G. I. Stegeman, A. A. Maradudin, and R. F. Wallis, J. Appl. Phys. 58, 2460 (1985).

[7] A. Boardman, V. Grimalsky, Y. Kivshar, S. Koshevaya, M. Lapine, N. Litchinitser, V. Malnev, M. Noginov, Y. Rapoport, and V. Shalaev, Laser Photon. Rev. 5, 287 (2011).

[8] M. Kauranen and A. V. Zayats, Nat. Photon. 6, 737 (2012).

[9] A. V. Zayats and S. Maier, Active Plasmonics and Tuneable Plasmonic Metamaterials (Wiley, New York, 2013), p. 336.

[10] Nonlinear, Tunable and Active Metamaterials, edited by I. V. Shadrivov, M. Lapine, and Y. S. Kivshar (Springer International Publishing, Berlin, 2015), Vol. 200, pp. XXII, 324.

[11] K. Leosson, J. Nanophoton 6, 061801 (2012).

[12] R. F. Oulton, V. J. Sorger, D. A. Genov, D. F. P. Pile, and X. Zhang, Nat. Photon. 2, 496 (2008).

[13] P. Berini, Adv. Opt. Photon. 1, 484 (2009).

[14] T. Holmgaard, J. Gosciniak, and S. Bozhevolnyi, Opt. Express 18, 23009 (2010).

[15] V. Volkov, Z. Han, M. Nielsen, K. Leosson, H. Keshmiri, J. Gosciniak, O. Albrektsen, and S. Bozhevolnyi, Opt. Lett. 36, 4278 (2011).

[16] M. P. Nezhad, K. Tetz, and Y. Fainman, Opt. Express 12, 4072 (2004).

[17] M. A. Noginov, V. A. Podolskiy, G. Zhu, M. Mayy, M. Bahoura, J. A. Adegoke, B. A. Ritzo, and K. Reynolds, Opt. Express 16, 1385 (2008).

[18] M. Ambati, S. Nam, E. Ulin-Avila, D. Genov, G. Bartal, and X. Zhang, Nano Lett. 8, 3998 (2008).

[19] J. Grandidier, G. des Francs, S. Massenot, A. Bouhelier, L. Markey, J. Weeber, C. Finot, and A. Dereux, Nano Lett. 9, 2935 (2009).

[20] I. De Leon and P. Berini, Nat. Photon. 4, 382 (2010).

[21] M. C. Gather, K. Meerholz, N. Danz, and K. Leosson, Nat. Photon. 4, 457 (2010).
[22] J. Zhang, L. Cai, W. Bai, Y. Xu, and G. Song, Opt. Lett. 36, 2312 (2011).

[23] S. García-Blanco, M. Pollnau, and S. Bozhevolnyi, Opt. Express 19, 25298 (2011).

[24] L. Gao, L. Tang, F. Hu, R. Guo, X. Wang, and Z. Zhou, Opt. Express 20, 11487 (2012).

[25] A. Marini, D. V. Skryabin, and B. Malomed, Opt. Express 19, 6616 (2011).

[26] C. Argyropoulos, N. Estakhri, F. Monticone, and A. Alù, Opt. Express 21, 15037 (2013).

[27] Z. Adelpour, A. Ghorbani, and V. Ahmadi, J. Opt. Soc. Am. B 31, 1672 (2014).

[28] E. Ding, A. Y. S. Tang, K. W. Chow, and B. A. Malomed, Philos. Trans. R. Soc. A 372, 20140018 (2014).

[29] B. A. Malomed, J. Opt. Soc. Am. B 31, 2460 (2014).

[30] A. Marini, S. Roy, A. Kumar, and F. Biancalana, Phys. Rev. A 91, 043815 (2015).

[31] S.-s. Wang, D.-q. Wang, X.-p. Hu, T. Li, and S.-n. Zhu, Chin. Phys. B 25, 077301 (2016).

[32] A. A. Sukhorukov, A. S. Solntsev, S. S. Kruk, D. N. Neshev, and Y. S. Kivshar, Opt. Lett. 39, 462 (2014).

[33] K. Y. Bliokh, Y. P. Bliokh, and A. Ferrando, Phys. Rev. A 79, 041803 (2009).

[34] C. Milián, D. E. Ceballos-Herrera, D. V. Skryabin, and A. Ferrando, Opt. Lett. 37, 4221 (2012).

[35] E. Feigenbaum and M. Orenstein, Opt. Lett. 32, 674 (2007).

[36] A. R. Davoyan, I. V. Shadrivov, and Y. S. Kivshar, Opt. Express 17, 21732 (2009).

[37] A. Marini, A. Gorbach, and D. Skryabin, Opt. Lett. 35, 3532 (2010).

[38] J. R. Salgueiro and Y. S. Kivshar, Appl. Phys. Lett. 97, 081106 (2010).

[39] F. Ye, D. Mihalache, B. Hu, and N. C. Panoiu, Phys. Rev. Lett. 104, 106802 (2010).

[40] W. Walasik, V. Nazabal, M. Chauvet, Y. Kartashov, and G. Renversez, Opt. Lett. 37, 4579 (2012).

[41] J. Salgueiro and Y. Kivshar, Opt. Express 20, 9403 (2012).

[42] P. Ginzburg, A. V. Krasavin, and A. V. Zayats, New J. Phys. 15, 013031 (2013).

[43] W. Walasik, G. Renversez, and Y. V. Kartashov, Phys. Rev. A 89, 023816 (2014).

[44] M. Sadeghi, V. Ahmadi, and M. Ebnali-Heidari, Plasmonics 11, 895 (2016). 
[45] W. Walasik and G. Renversez, Phys. Rev. A 93, 013825 (2016).

[46] W. Walasik, G. Renversez, and F. Ye, Phys. Rev. A 93, 013826 (2016).

[47] A. Ferrando, C. Milián, and D. V. Skryabin, J. Opt. Soc. Am. B 30, 2507 (2013).

[48] Y. Eksioglu, O. E. Müstecaplioglu, and K. Güven, Phys. Rev. A 84, 033805 (2011).

[49] Y. Eksioglu, O. E. Müstecaplioglu, and K. Güven, Phys. Rev. A 87, 023823 (2013).

[50] $N_{p}$ is the plasmon norm of the unperturbed system, which is complex in the case of a lossy metal [see Eq. (A11)]. However, its imaginary part is smaller than the real part and $N_{p}^{\prime \prime}=\operatorname{Im}\left(N_{p}\right) \rightarrow$ 0 when $\operatorname{Im}\left(\varepsilon_{m}\right) \rightarrow 0$ so $N_{p}^{\prime \prime}$ can be considered a perturbation. Since $\delta \varepsilon_{p}$ is itself a perturbation, we can approximate $N_{p}$ by its real part at first order.

[51] M. Chauvet, G. Fanjoux, K. P. Huy, V. Nazabal, F. Charpentier, T. Billeton, G. Boudebs, M. Cathelinaud, and S.-P. Gorza, Opt. Lett. 34, 1804 (2009).

[52] N. Akhmediev and A. Ankiewicz, Dissipative Solitons: From Optics to Biology and Medicine (Lecture Notes in Physics) (Springer, New York, 2008), p. 479.
[53] P. W. Milonni and J. H. Eberly, Lasers Physics (John Wiley \& Sons, Hoboken, NJ, 2010), pp. xiv, 830.

[54] L. Novotny, Am. J. Phys. 78, 1199 (2010).

[55] Z. H. Musslimani, K. G. Makris, R. El-Ganainy, and D. N. Christodoulides, Phys. Rev. Lett. 100, 030402 (2008).

[56] S. V. Suchkov, A. A. Sukhorukov, J. Huang, S. V. Dmitriev, C. Lee, and Y. S. Kivshar, Laser Photon. Rev. 10, 177 (2016).

[57] S. Suchkov, B. Malomed, S. Dmitriev, and Y. Kivshar, Phys. Rev. E 84, 046609 (2011).

[58] D. A. Zezyulin and V. V. Konotop, Phys. Rev. Lett. 108, 213906 (2012).

[59] P. G. Kevrekidis, D. E. Pelinovsky, and D. Y. Tyugin, J. Phys. A 46, 365201 (2013).

[60] K. Li, P. G. Kevrekidis, and B. A. Malomed, Stud. Appl. Math. 133, 281 (2014).

[61] B. A. Malomed and H. G. Winful, Phys. Rev. E 53, 5365 (1996).

[62] J. Atai and B. A. Malomed, Phys. Lett. A 246, 412 (1998).

[63] H. Nistazakis, D. Frantzeskakis, J. Atai, B. Malomed, N. Efremidis, and K. Hizanidis, Phys. Rev. E 65, 036605 (2002).

[64] L. Mandel and E. Wolf, Optical Coherence and Quantum Optics (Cambridge University Press, Cambridge, UK, 1995), p. 1192. 